\title{
山竹醇新类似物的合成及其抗肿瘤活性研究
}

\author{
周汇源 ${ }^{a}$ 吴堰霖 $b$ 黄典红 ${ }^{a}$ 张铭婷 ${ }^{a}$ 陈欢明 ${ }^{a}$ \\ 钱明成 $a$ 赵帅 $a$ 张辛燕*,b 陈 新*, $a$ \\ ( ${ }^{a}$ 常州大学制药与生命科学学院 江苏常州 213164) \\ $\left({ }^{b}\right.$ 首都医科大学附属北京口腔医院 口腔医学研究所 北京 100050)
}

\begin{abstract}
摘要 山竹醇具有广泛的生物学活性, 例如抗炎、抗肿瘤、抗氧化、诱导细胞调亡等. 通过对山竹醇进行结构修饰, 研 究其侧链基团对山竹醇活性的影响, 以期提高其抗肿瘤活性. 以乙酰丙酮为原料, 经过 Michael 加成、Knoevenagel 缩 合等多步反应, 合成了三个未见文献报道的山竹醇类似物, 并用噻桽蓝(MTT)法对其进行生物活性研究, 分析其构效 关系. 结果表明, 合成的三种新类似物对口腔鳞癌细胞的抑制活性均低于山竹醇, 由此可知, C4 位置的异戊烯基和 C8 位置的烯丙基对山竹醇的生物活性起关键作用.
\end{abstract}

关键词 山竹醇; 结构修饰; Michael 加成; 抗肿瘤

\section{Synthesis and Anti-tumor Activity of Novel Garcinol Analogs}

\author{
Zhou, Huiyuan $^{a} \quad \mathrm{Wu}$, Yanlin $^{b}$ \\ Huang, Dianhong ${ }^{a}$ \\ Zhang, Mingting ${ }^{a}$ \\ Chen, Huanming ${ }^{a}$ \\ Qian, Mingcheng ${ }^{a}$ \\ Zhao, Shuai $^{a}$ \\ Zhang, Xinyan*,b \\ Chen, $\mathrm{Xin}^{*, a}$ \\ ( ${ }^{a}$ School of Pharmaceutical Engineering and Life Science, Changzhou University, Changzhou, Jiangsu 213164) \\ ( ${ }^{b}$ Beijing Institute of Dental Research, Beijing Stomatological Hospital, Capital Medical University, Beijing 100050)
}

\begin{abstract}
Garcinol possesses a wide range of biological activities, such as anti-inflammation, anti-tumors, anti-oxidation, induction of apoptosis and so on. In this paper, the modification of the side chains in garcinol was carried out to enhance its anti-tumor activity. Employing acetylacetone as starting material, three new analogs of garcinol were prepared by means of Michael addition, Knoevenagel condensation, and so on. Furthemore, their biological activity was assessed by methyl thiazolyl tetrazolium (MTT) method and the structure-activity relationship was analyzed. The results showed that the inhibitory activities of the three new analogs on oral squamous cell carcinoma were moderately lower than that of garcinol. Hence, the isoprenyl group at the $\mathrm{C} 4$ position and the allylic group at the $\mathrm{C} 8$ position might play vital roles for the biological activity of garcinol.
\end{abstract}

Keywords garcinol; structural modification; Michael addition; anti-tumor

天然产物是新药的重要来源, 特别是在抗肿瘤和降 血压领域 ${ }^{[1]}$. 在印度, 乳香、姜黄、白生丹、藤黄等天然 植物或从中提取的化合物, 已经被证明具有非常高的药 用价值 ${ }^{[2,3]}$. 山竹醇 (Garcinol, 图 1), 黄色晶体化合物, 是从印度藤黄的干果皮中提取出来的一种多聚异戊二 烯基苯甲䣯 ${ }^{[4]}$. 大量研究表明, 山竹醇具有广泛的生物 学活性, 例如抗炎、抗氧化、抗增殖、诱导细胞凋亡等, 主要是由于山竹醇能抑制 5-脂氧化酶(5-LOX)和环氧化 酶-2 (COX-2)的活性 ${ }^{[\sim 11]}$.

近年来, 山竹醇及其衍生物对抑制口腔鳞癌细胞的
活性引起了人们的研究兴趣 ${ }^{[12 \sim 14]}$. 我们课题组前期通 过计算机建模预测，山竹醇与 5-LOX 的活性位点相结 合，从而抑制 5-LOX ${ }^{[15]}$. 而山竹醇结构中 $\mathrm{C} 8$ 位置上的 过大支链可能会阻碍山竹醇与 5-LOX 的活性位点相结 合，导致不能有效抑制 5-LOX. 我们近期的研究表明， 将 $\mathrm{C} 8$ 位置的侧链换成更小的甲基，即 8-甲基山竹醇 ${ }^{[12]}$, 抗肿瘤活性比山竹醇弱. 而将 C8 位置侧链换成烯丙基 时 ${ }^{[13]}$, 所生成 8 -烯丙基山竹醇在中低浓度 $(5-20 \mu \mathrm{mol} \cdot$ $\mathrm{L}^{-1}$ ) 时, 抑制效果优于山竹醇. 这些结果表明, C8 位置 的不饱和侧链是其活性必需基团. 同时, 我们也证明了

* Corresponding authors. E-mail: xinyanzhangzh@126.com; xinchen@cczu.edu.cn Received January 9, 2020; revised March 9, 2020; published online April 10, 2020 Project supported by the National Natural Science Foundation of China (Nos. 21272029, 81772868). 国家自然科学基(Nos. 21272029, 81772868)资助项目. 
山竹醇 13 和 14 位两个羟基是其抗肿瘤活性的必需基 团 $^{[14]}$. 在此基础上, 我们继续对山竹醇的结构进行修 饰, 计划将 C8 位置的九碳取代基替换为异戊烯基, 简 称为 8-异戊烯基(代)山竹醇 [8-isoprenyl garcinol, 全称: 8-异戊烯基-8-去-(2-异丙烯基-5-甲基-己-4-烯基)山竹 醇](1)(图 1). 并且, 我们还将山竹醇母体桥环结构中桥 头的另一端进行修饰, 即将 $\mathrm{C} 4$ 和 $\mathrm{C} 8$ 位置的取代基替换 成烯丙基, 简称 4,8-二烯丙基(代)山竹醇(4,8-diallyl garcinol, 全称：4,8-双(烯丙基)-4-去-(异戊烯基)-8-去-(2-异 丙烯基-5-甲基-己-4-烯基)-山竹醇)(2). 将 C4 位置取代 基替换为烯丙基, $\mathrm{C} 8$ 位置取代基替换为异戊烯基，简称 4-烯丙基-8-异戊烯基(代)山竹醇(4-allyl-8-isoprenyl garcinol, 全称：4-烯丙基-4-去-(异戊烯基)-8-异戊烯基-8-去 -(2-异丙烯基-5-甲基-己-4-烯基)-山竹醇)(3).
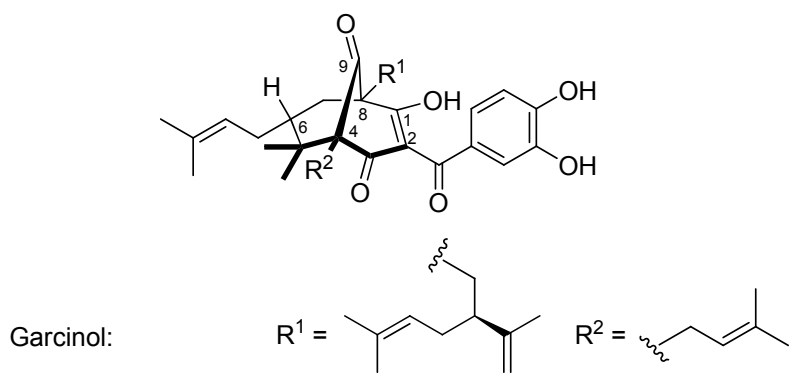

1: 8-isoprenyl Garcinol: $\quad R^{1}=R^{2}=$

2: 4,8-diallyl Garcinol: $\quad \mathrm{R}^{1}=\mathrm{R}^{2}=$

3: 4-allyl-8-isoprenyl Garcinol: $\mathrm{R}^{1}=$

图 1 山竹醇及其新类似物的化学结构

Figure 1 Structures of garcinol and novel garcinol analogs

目前, 山竹醇及其衍生物的合成研究日渐引起人们 的兴趣. Socolsky 等 ${ }^{[16]}$ 以乙酰丙酮作为起始原料, 经过 Michael 加成, Knoevenagel 缩合, Dieckmann 缩合等多步 反应合成得到山竹醇. 2017 年, Guttroff 等 ${ }^{[17]}$ 用类似的方 法, 经过多步反应合成了多种山竹醇结构类似物. 在本 文中, 我们使用上述类似的合成方法, 成功地合成了三 种未见报道的山竹醇类似物 $1 \sim 3$, 并对其进行抗肿瘤 活性篮选.

\section{1 结果与讨论}

\section{1 化学合成部分}

参照 Biber 等 ${ }^{[18]}$ 的方法, 以乙酰丙酮(4)为起始原料 合成了山竹醇的三个新类似物. 如 Scheme 1 所示, 在 $\mathrm{NaH}$ 作用下, 4 与 3,3-二甲基烯丙基溴进行烯丙基化反 应，随后进行 $\alpha$ 亚甲基化得到中间产物 $\mathbf{5}^{[18]}$, 然后, $\mathbf{5}$ 与
1,3-丙酩二羧酸二甲酯发生 Michael 加成和 Knoevenagel 缩合串联反应，并进行环化，以 $89 \%$ 产率得到消旋化中 间体 6. 随后甲基锂对中间体 6 进行 1,2-加成生成相应 的甲基酮类化合物，然后再进行羰基 $\alpha$ 位烯丙基化，以 较高的产率和高非对映选择性得到化合物 7, 其高选择 性主要原因是处于坚键的异戊烯基阻挡了亲电试剂从 烯醇平面下方的进攻 ${ }^{[18]}$. 此步反应要注意的是, 氢化钠 需要分批加入, 温度要控制在 $0{ }^{\circ} \mathrm{C}$, 否则温度过高, 烷 基化试剂会在其他位置发生取代反应. 接着, 化合物 7 与甲基酮锂进行 Michael 加成, 得到双甲基化产物 8. 为 了在 8 中两个羰基之间引入异戊烯基，起初使用了第三 步的条件, 即以 $\mathrm{NaH}$ 作为碱, 8 与异戊烯基溴反应, 主要 得到的却是 $O$-烯丙基化产物. 后来, 使用 Plietker 小组 报道 ${ }^{[17,18]}$ 的含 $\mathrm{Fe}$ 催化剂 $\mathrm{Bu}_{4} \mathrm{~N}\left[\mathrm{Fe}(\mathrm{CO})_{3}(\mathrm{NO})\right]^{[19]}$ 和配体 SIMES* $\mathrm{PF}_{6}{ }^{[20]}$ 后，则顺利得到了 $C$-烯丙基化产物 9. 在 叔丁醇钾的作用下，中间体 9 进行 Dieckmann 缩合得到 三环中间体 10. 为了得到目标化合物 1 , 需要对 10 进行 酰化反应. 根据本实验室前期的研究 ${ }^{[13]}, 3,4$-二乙酰氧 基苯甲酰氰比相应的酰氯更好地与 10 反应，生成酰基 化产物 11. 最后, 在 $\mathrm{K}_{2} \mathrm{CO}_{3}$ 和甲醇溶液中，化合物 $\mathbf{1 1}$ 发 生水解得到目标化合物 $\mathbf{1}$.

对于中间体 9 的形成过程, Scheme 2 参照 Biber 等 ${ }^{[18]}$ 的解释进行了说明. 在含 $\mathrm{Fe}$ 催化剂 $\mathrm{Bu}_{4} \mathrm{~N}\left[\mathrm{Fe}(\mathrm{CO})_{3}{ }^{-}\right.$ (NO)] 和配体 $\mathrm{SIMES}^{*} \mathrm{PF}_{6}$ 作用下, 8 与 2-甲基-3-丁烯-2碳酸异丁酯反应，在所形成的可能中间体中，由于坚键 位的羧酸甲酯 $(\mathrm{C} 1$ 位)和甲基 $(\mathrm{C} 4$ 位)阻挡了亲电试剂从 烯醇平面下方的进攻，新引入的异戊烯基选择性地从烯 醇平面上方进攻 2 位碳原子 $\left(\mathrm{R}=\mathrm{R}^{\prime}=\right.$ 异戊烯基, $\mathrm{Met}=$ 金 属离子), 从而得到 $\beta$-构型为主的加成产物 9.

山竹醇类似物 2 和 3 的合成如 Scheme 3 所示. 为了 在 8 和 13 中两个羰基之间引入烯丙基, 8 和先与氯甲酸 烯丙基酯反应，分别得到区域异构体混合物 $8 \mathrm{a} \sim 8 \mathrm{~b}$ 和 13a 13b. 随后在 Pd 催化剂作用下发生 Tsuji-Trost 反 应 $^{[17]}$ ，以较高的产率得到主要产物 14 和 17 . 接着，中间 体 14 和 17 发生 Dieckmann 缩合分别得到环化产物 15 和 18 , 然后酰基化得到中间体 16 和 19 , 最后水解得到 山竹醇类似物 $\mathbf{2}$ 和 3.

\section{2 山竹醇及其类似物的抗肿瘤活性评价}

我们利用 MTT 法, 测试了山竹醇和山竹醇新类似 物(1, 2 和 3) 对口腔鳞癌 SCC-15 和 CAL-27 细胞的抗增 殖活性. 结果表明, 与阴性对照组相比, 山竹醇和山竹 醇新类似物均能够抑制口腔鳞癌细胞 SCC-15 和 CAL-27 的增殖, 差异具有统计学意义 $(P<0.01)$. 图 2 中，我们可以看到孵育时间不同，不同化合物的活性大 小顺序有变化, 而图 3 中的活性大小顺序几乎无变化. 


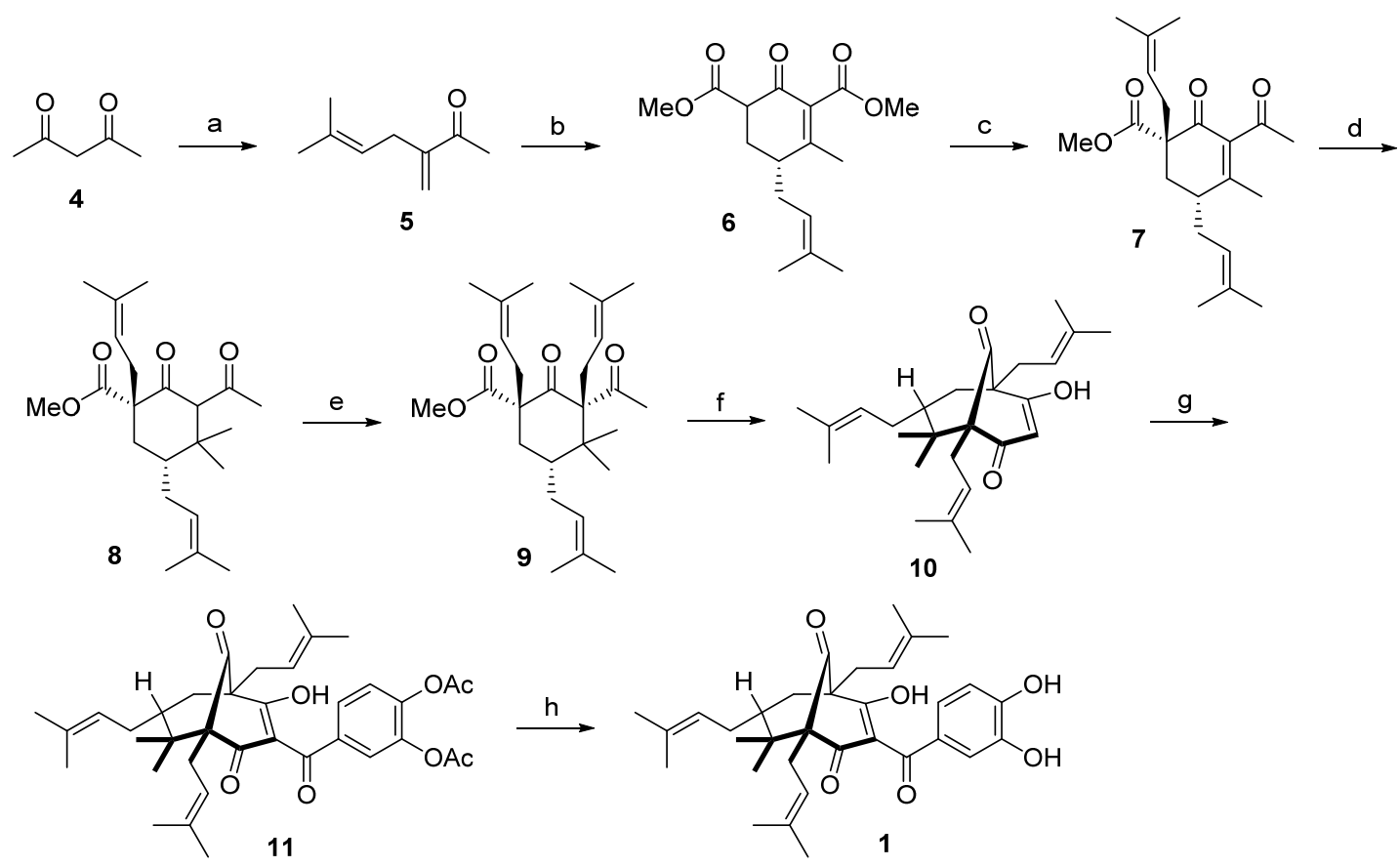

Reagents and conditions: (a) $\mathrm{NaH}$, isoprenyl bromide, $\mathrm{EtOH}, 0{ }^{\circ} \mathrm{C}$ to r.t., $15 \mathrm{~h}$, then $\mathrm{K}_{2} \mathrm{CO}_{3}$, formaldehyde, r.t., $15 \mathrm{~h}, 60 \%$ yield. (b) methylmagnesium chloride, dimethyl 1,3-acetonedicarboxylate, $\mathrm{MeOH}, 0$ to $60{ }^{\circ} \mathrm{C}, 15 \mathrm{~h}, 89 \%$ yield. (c) $\mathrm{NaH}$, THF, then MeLi, $0{ }^{\circ} \mathrm{C} ; \mathrm{NaH}$, isoprenyl bromide, THF, $0{ }^{\circ} \mathrm{C}$ to r.t., $63 \%$ yield. (d) $\mathrm{LiCl}$, Cul, methylmagnesium bromide, $\mathrm{Me}_{3} \mathrm{SiCl}$, THF, $-78{ }^{\circ} \mathrm{C}, 96 \%$ yield. (e) Potassium tert-amylate $\left(\mathrm{KO}^{\dagger} \mathrm{Am}\right)$, 1,3-dimesitylimidazolin-2-ylidene hexafluorophosphate $\left(\mathrm{SIMES}^{*} \mathrm{PF}_{6}\right)(10 \mathrm{~mol} \%), \mathrm{Bu}_{4} \mathrm{~N}\left[\mathrm{Fe}(\mathrm{CO})_{3}(\mathrm{NO})\right](10 \mathrm{~mol} \%)$, LiH, isobutyl (2-methylbut-3-en-2-yl) carbonate, THF/methyl-t-butyl ether, 0 to $80{ }^{\circ} \mathrm{C}, 44 \%$ yield. (f) $\mathrm{KOBu}^{t}, \mathrm{THF}, 0{ }^{\circ} \mathrm{C}, 80 \%$ yield. (g) Et ${ }_{3} \mathrm{~N}, 4-(\mathrm{cyanocar}-$ bonyl)-1,2-phenylene diacetate, THF, r.t., $16 \mathrm{~h}, 76 \%$ yield. (h) $\mathrm{K}_{2} \mathrm{CO}_{3}, \mathrm{MeOH}$, r.t., $1 \mathrm{~h}, 64 \%$ yield.

图式 1 山竹醇类似物 1 的合成

Scheme 1 Synthesis of garcinol analog 1<smiles>COC(=O)[C@]1(CC(C)=O)C[C@H](CC=C(C)C)C(C)(C)C(C(C)=O)C1=O</smiles>

8

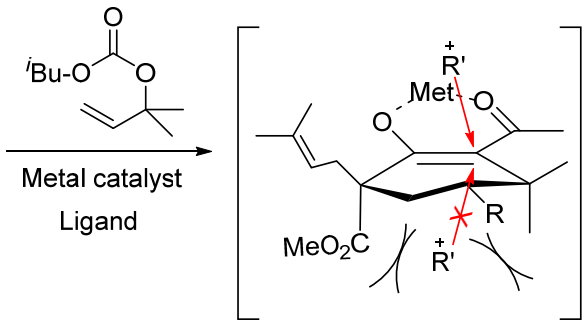

$\mathrm{R}=\mathrm{R}^{\prime}=$ isoprenyl, Met: metal catalyst

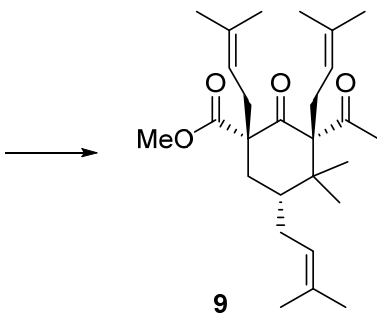

9

图式 2 中间体 9 的形成机理

Scheme 2 Formation of intermediate 9

这主要是因为化合物的活性与其分子量大小, 在细胞内 代谢的速度以及对细胞膜的穿透能力都有很大关系, 并 且细胞系的不同, 化合物的活性大小也会不同. 总体来 说, 随着山竹醇和山竹醇新类似物浓度的增高和作用时 间延长, 对 SCC-15 和 CAL-27 细胞的抑制作用逐渐增 强, 呈一定的浓度和时间依赖关系(图 2 和 3). 但是, 与 山竹醇对比, 山竹醇新类似物 $(1,2$ 和 3$)$ 的抗增殖作用均 不同程度的低于山竹醇.

根据之前的研究 ${ }^{[14]}, 8$-烯丙基山竹醇的抑制作用在 低至中浓度优于山竹醇. 在 8-烯丙基山竹醇的研究基础 上, 将 C8 位置的烯丙基换成空间需求更大的异戊烯基
时，即 8-异戊烯基山竹醇，抑制作用较山竹醇明显降低. 当把 C4 位置的异戊烯基替换成烯丙基时，即 4,8-二烯 丙基山竹醇，同样，抑制作用较山竹醇降低. 接着，将 C4 位置替换成烯丙基并且 C8 位置替换成异戊烯基，即 4-烯丙基-8-异戊烯基山竹醇, 抗增殖活性相较于山竹醇 同样不理想. 根据活性测试结果，我们猜测 C4 和 C8 位 置的侧链可能对山竹醇的抗增殖活性有影响.

\section{3 山竹醇及其类似物与 5-LOX 的分子对接}

为了进一步阐释山竹醇及其类似物的抗肿瘤活性 结果，我们通过 Discovery Studio 2019 Client (DS 2019) 软件, 将山竹醇及其类似物分别与 5-LOX 进行分子对 


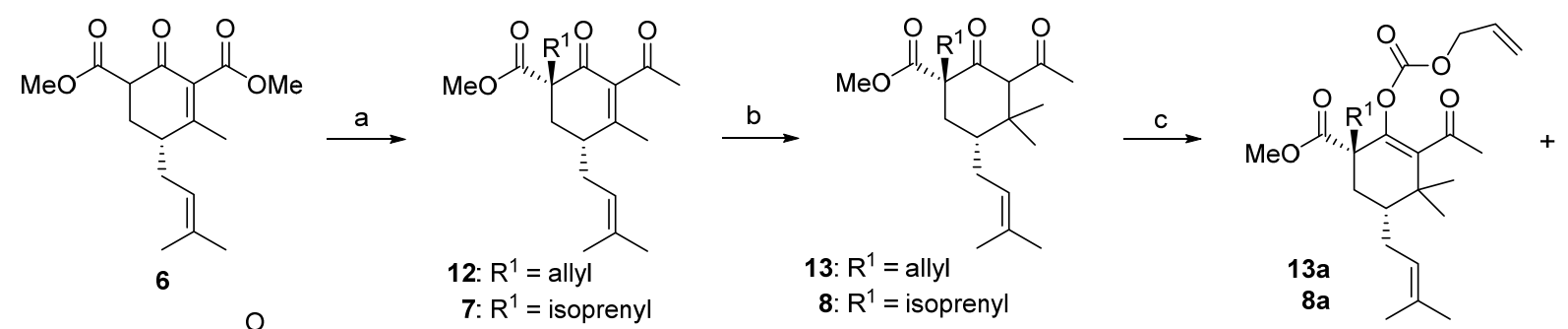

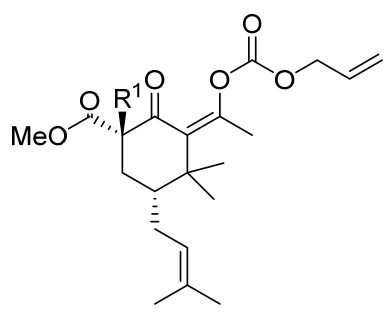

13b: $R^{1}=$ allyl 8b: $R^{1}=$ isoprenyl<smiles>COC(=O)[C@]1(Br)C[C@H](CC=C(C)C)C(C)(C)[C@@](C(C)=O)(C(=O)OC)C1=O</smiles>

14: $R^{1}=$ allyl<smiles>[R]C12C(=O)C3(C(=O)C(C(=O)c4ccc(OC(C)=O)c(OC(C)=O)c4)=C(O)C31[R])C(CC=C)C2(C)CC=C(C)C</smiles>

16: $R^{1}=$ allyl

19: $R^{1}=$ isoprenyl

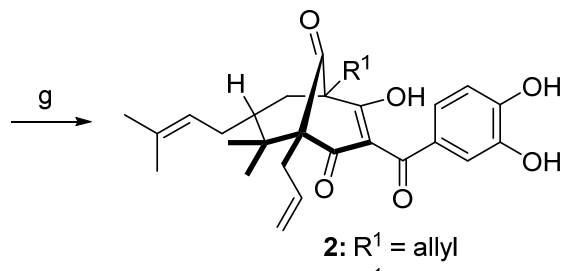

3: $\mathrm{R}^{1}=$ isoprenyl

Reagents and conditions: (a) NaH, THF, then MeLi, $0{ }^{\circ} \mathrm{C}$; NaH, $\mathrm{R}^{1} \mathrm{Br}, 0{ }^{\circ} \mathrm{C}$ to r.t., for 7: $63 \%$ yield, for 12: $66 \%$ yield; (b) LiCl, Cul, methylmagnesium bromide, $\mathrm{Me}_{3} \mathrm{SiCl}, \mathrm{THF},-78{ }^{\circ} \mathrm{C}$. For 8: $96 \%$ yield; for $13: 91 \%$ yield; (c) $\mathrm{NaH}$, allyl chloroformate, DMF, $0{ }^{\circ} \mathrm{C}$ to r.t., for $8 \mathrm{a}$ : $34 \%$ yield, 8b: $50 \%$ yield; for 13a: $32 \%$ yield, 13b: $63 \%$ yield; (d) Tris(dibenzylideneacetone)dipalladium(0)-chloroform $\left[\mathrm{Pd}_{2}\left(\mathrm{dba}_{3}-\mathrm{CHCl}_{3}\right](5 \mathrm{~mol} \%)\right.$, tri( $p$-tolyl)phosphine, toluene, r.t., for $14: 73 \%$ yield, for $17: 75 \%$ yield. (e) $\mathrm{KOBu}^{t}$, THF, $0{ }^{\circ} \mathrm{C}$, for $15: 81 \%$ yield, for 18 : $72 \%$ yield; (f) $\mathrm{Et}_{3} \mathrm{~N}$, 4-(cyanocarbonyl)-1,2-phenylene diacetate, THF, r.t., $16 \mathrm{~h}$, for $16: 78 \%$ yield, for $19: 75 \%$ yield; $(\mathrm{g}) \mathrm{K}_{2} \mathrm{CO}_{3}, \mathrm{MeOH}$, r.t., $1 \mathrm{~h}$, for $2: 69 \%$ yield, for 3: $58 \%$ yield.

图式 3 山竹醇类似物 $\mathbf{2}$ 和 3 的合成

Scheme 3 Synthetic of garcinol analogs 2 and 3
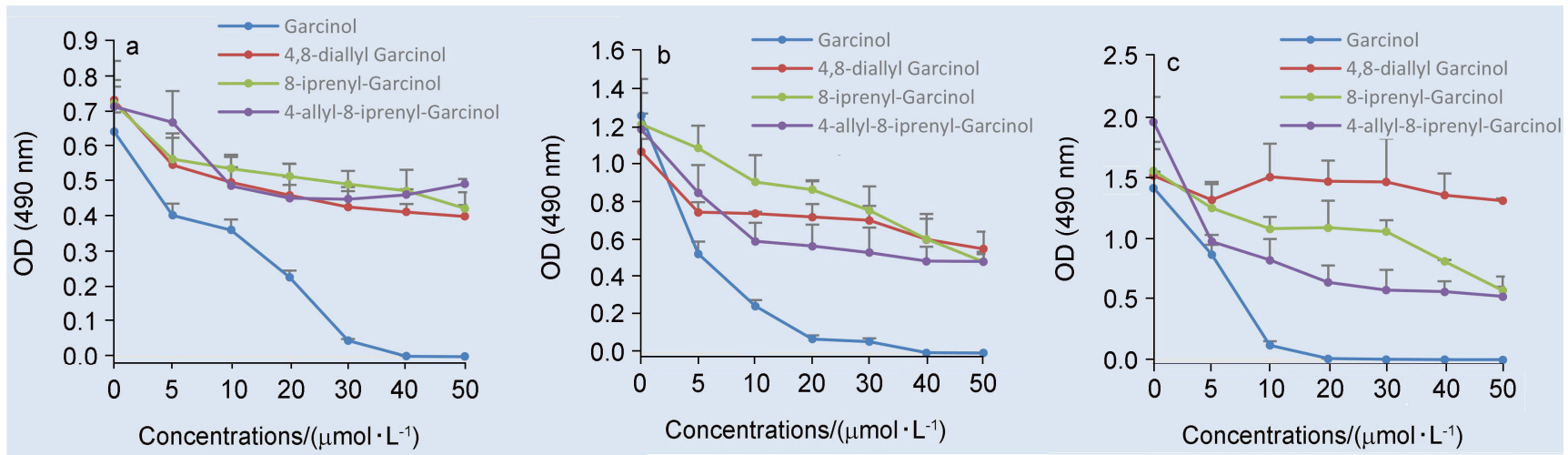

图 2 不同浓度山竹醇和山竹醇类似物处理 CAL-27 细胞 24 (a)、48 (b) 和 $72 \mathrm{~h}$ (c) 的 OD 值

Figure 2 OD values of CAL-27 cells treated with different concentrations of garcinol and garcinol analogs at 24 (a), 48 (b) and 72 h (c)

接. 分子对接结果显示, CDOCKER Energy 的数值全部 为负数, 表明分子内能较高. 本实验中, 我们采用CDOCKER Interaction Energy 作为打分函数判定依据, 其数值分别为: 山竹醇: 69.8055 , 类似物 1: 64.8402, 类 似物 2: 53.0178, 类似物 3: 60.4108. 分子模拟结果表明, 相比于山竹醇的三个类似物, 山竹醇与 5-LOX 结合位 点相互作用时所需要的能量最低.
图 4 是山竹醇及其类似物与 $5-\mathrm{LOX}$ 分子对接 2D 图. 山竹醇与 5-LOX 结合位点处的 Glu614、His550、His367、 Leu607 和 Phe177 等氨基酸残基产生非键作用: Glu614 与苯环上两个羟基形成氢键; Phe 555 与苯环有 $\pi-\pi$ 堆积 作用; 连接苯环的 $\mathrm{C}=\mathrm{O}$ 与金属受体 FE2701 结合, 同时 与 His550 产生碳氢键作用; His367 与 $\mathrm{C} 1$ 位置的 $\mathrm{O}$ 产生 氢键作用. 类似物 1 与 5-LOX 结合位点处的 Phe610、 


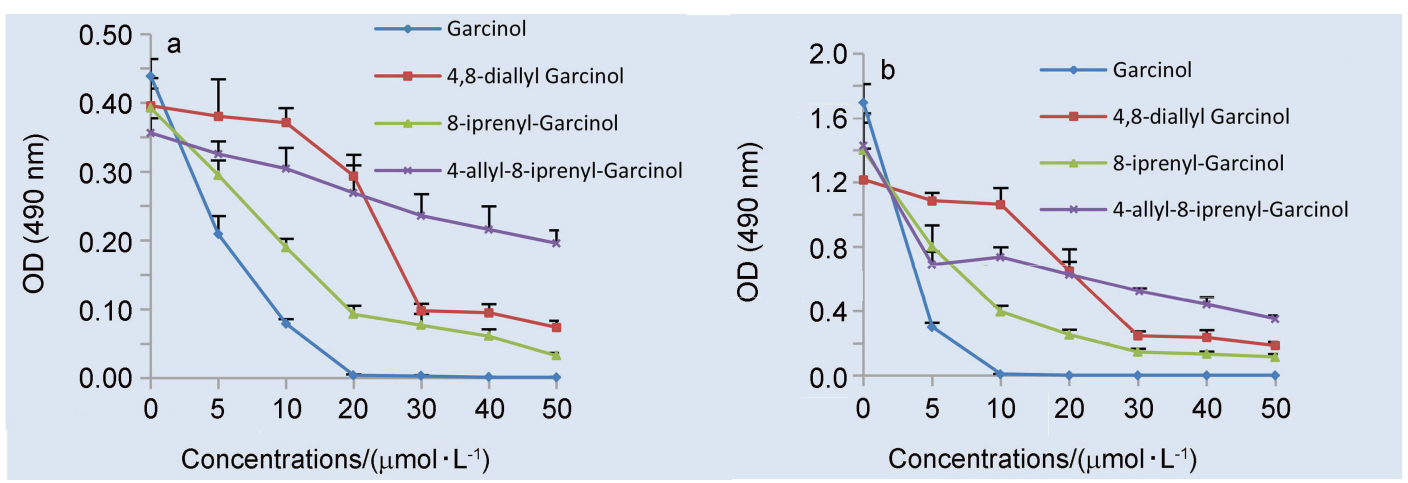

图 3 不同浓度山竹醇和山竹醇类似物处理 SCC-15 细胞 24 (a) 和 $72 \mathrm{~h}(\mathrm{~b})$ 的 OD 值

Figure 3 OD values of SCC-15 cells treated with different concentrations of garcinol and garcinol analogs at 24 and $72 \mathrm{~h}$
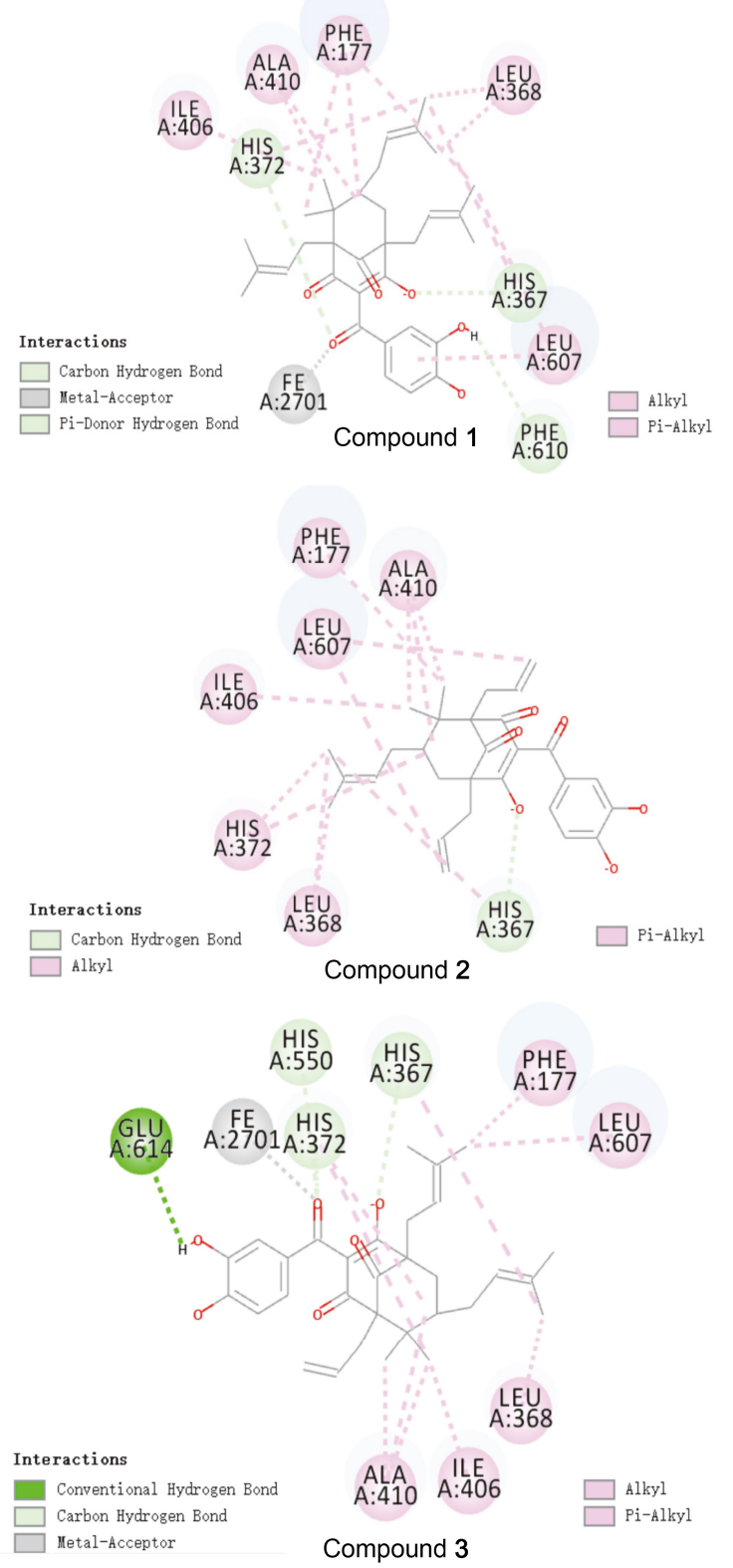

图 4 山竹醇及其类似物 1,2 和 3 与 $5-\mathrm{LOX}$ 分子对接 $2 \mathrm{D}$ 图 Figure 4 Molecular docking 2D modeling of garcinol and garcinol analogs 1, 2 and $\mathbf{3}$ with 5-LOX
His372、His367 和 Leu607 等氨基酸残基产生非键作用: Phe610 与苯环上一个羟基发生氢键作用; 连接苯环的 $\mathrm{C}=\mathrm{O}$ 与金属受体 FE2701 结合，同时与 His372 产生碳 氢键作用; His 367 与 $\mathrm{C} 1$ 位置的 $\mathrm{O}$ 形成氢键作用. 类似物 2 与 5-LOX 结合位点处的 His367、Leu368、His372 和 Phe177 等氨基酸残基产生非键作用: His 367 与 $\mathrm{C} 1$ 位置 的 $\mathrm{O}$ 形成氢键作用. 类似物 3 与 5-LOX 结合位点处的 Glu614、His550、His367、His372 和 Phe177 等氨基酸 残基产生非键作用: Glu614 与苯环上一个羟基产生氢键 作用; 连接苯环的 $\mathrm{C}=\mathrm{O}$ 与金属受体 $\mathrm{FE} 2701$ 结合，同时 与 His550、His372 产生氢键作用; His367 与 C1 位置的 $\mathrm{O}$ 形成氢键作用.

综上所述，DS 模拟结果表明，与山竹醇类似物相 比, 山竹醇苯环上两个酚羟基分别与 5-LOX 结合位点 处的 Glu614 形成两个氢键相互作用. 因此, 山竹醇与 5-LOX 的相互作用能相比于新类似物(1, 2 和 3)都比较 强. 分子模拟结果和细胞抗增殖结果一致, 为山竹醇的 结构优化提供了思路.

\section{2 结论}

为了提高山竹醇对肿瘤细胞的抑制活性，并研究其 构效关系，本文以乙酰丙酮为原料，设计并合成了三个 山竹醇的新类似物. MTT 实验结果表明，新合成的山竹 醇类似物对人口腔鳞癌细胞的抗增殖活性均不同程度 弱于山竹醇; 构效关系研究表明, 山竹醇 C4 和 C8 位的 侧链对山竹醇的抗增殖活性具有重要作用, 这些结果对 后续山竹醇的结构优化与改造研究具有重要的指导意 义.

\section{3 实验部分}

\section{1 仪器与试剂}

熔点使用上海申光公司的显微熔点仪, 型号为 SGW X-4B; NMR 用 Bruker (400 和 $500 \mathrm{MHz}$ )型核磁共 振仪测定 $\left(\mathrm{CDCl}_{3}, \mathrm{CD}_{3} \mathrm{OD}\right.$ 作溶剂, TMS 为内标); HRMS 
用 Thermo Orbitrap Elite 测定; 昆山市超声仪器有限公 司 SQ-5200 型超声波清洗器; 分子对接模拟使用 Discovery Studio 2019 Client (DS 2019)软件; 150 200 目硅胶(青岛海洋化工厂生产); 薄层色谱使 HSGF-254 (购自烟台江友硅胶开发有限公司). 试剂均为分析纯. $\mathrm{Fe}$ 催化剂 $\mathrm{Bu}_{4} \mathrm{~N}\left[\mathrm{Fe}(\mathrm{CO})_{3}(\mathrm{NO})\right]$ 按照文献[19]方法合成, 配体 SIMES*PF 6 按照文献[20]方法合成.

\section{2 实验方法}

\subsubsection{6-甲基-3-亚甲基庚-5-烯-2-酮(5)的合成}

将乙酰丙酮 $(20 \mathrm{~g}, 199.7 \mathrm{mmol}$ )溶于乙醇 $(140 \mathrm{~mL})$ 中, 冷却至 $0{ }^{\circ} \mathrm{C}$, 分批加入 $\mathrm{NaH}(60 \%$ in mineral oil, $8.8 \mathrm{~g}$, $219.7 \mathrm{mmol}$ ), 搅拌 $5 \mathrm{~min}$ 后, 缓慢滴加 3,3-二甲基烯丙 基溴(32.8 mL, $299.6 \mathrm{mmol}$ ), 然后将反应液升至室温, 搅拌过夜. 随后将 $37 \%$ 甲醛水溶液 $(50 \mathrm{~mL})$ 和 $\mathrm{K}_{2} \mathrm{CO}_{3}$ $(55.2 \mathrm{~g}, 399.4 \mathrm{mmol}$, 溶于 $200 \mathrm{~mL}$ 水)加入反应液, 室温 搅拌过夜, 薄层色谱(TLC)监测反应进程. 反应毕, 向反 应液加入 $280 \mathrm{~mL}$ 水, 混合物用乙酸乙酯萃取 (300 mL × 3), 合并有机相, 再经过饱和食盐水洗涤, 无水硫酸钠 干燥, 过滤浓缩, 并进行柱层析纯化, 得到无色液体 $5^{[18]}\left(16.5 \mathrm{~g}\right.$, 产率 60\%). ${ }^{1} \mathrm{H}$ NMR $\left(400 \mathrm{MHz}, \mathrm{CDCl}_{3}\right) \delta$ : $5.97(\mathrm{~s}, 1 \mathrm{H}), 5.71(\mathrm{~s}, 1 \mathrm{H}), 5.11 \sim 5.07(\mathrm{~m}, 1 \mathrm{H}), 2.90(\mathrm{~d}, J=$ $7.3 \mathrm{~Hz}, 2 \mathrm{H}), 2.31$ (s, 3H), $1.69(\mathrm{~s}, 3 \mathrm{H}), 1.57(\mathrm{~s}, 3 \mathrm{H}) ;{ }^{13} \mathrm{C}$ NMR $\left(100 \mathrm{MHz}, \mathrm{CDCl}_{3}\right) \delta: 199.8,148.1,134.0$ 125.0, $120.8,29.0,26.0,25.8,17.6$.

3.2.2（土）-4-甲基-5-(3-甲基丁-2-烯-1-基)-2-氧亚基 环已基-3-烯-1,3-二羧酸二甲酯(6)的合成

取 $115 \mathrm{~mL}$ 甲醇于烧瓶中, $0{ }^{\circ} \mathrm{C}$ 下滴加甲基氯化镁(3 $\mathrm{mol} \cdot \mathrm{L}^{-1}$ in THF, $38.6 \mathrm{~mL}, 115.8 \mathrm{mmol}$ )和 1,3 -丙酮二甲酸 二甲酯 $(4.7 \mathrm{~g}, 57.9 \mathrm{mmol})$, 并在该温度下搅拌 $1 \mathrm{~h}$. 随后 将化合物 $2(8 \mathrm{~g}, 57.9 \mathrm{mmol})$ 滴加至反应液中, $60{ }^{\circ} \mathrm{C}$ 搅拌 回流过夜, TLC 监测. 反应毕, 滴加 $2 \mathrm{~mol} / \mathrm{L} \mathrm{HCl}(42$ $\mathrm{mL}$ ), 减压浓缩有机相, 水相用乙酸乙酯萃取 (50 mL $\times$ 3), 合并有机相, 有机相用水洗, 饱和食盐水洗, 无水 硫酸钠干燥, 减压浓缩, 粗产物通过柱层析分离化 [洗 脱剂: $V$ (石油醚) $: V($ 乙酸乙酯 $)=8: 1$ ] , 得到淡黄色油 状物 6 (15.1 g, 产率 89\%). ${ }^{1} \mathrm{H}$ NMR (400 MHz, $\left.\mathrm{CDCl}_{3}\right) \delta$ : $5.09 \sim 5.04(\mathrm{~m}, 1 \mathrm{H}), 3.78(\mathrm{~s}, 3 \mathrm{H}), 3.72(\mathrm{~s}, 3 \mathrm{H}), 3.52 \sim 3.48$ $(\mathrm{m}, 1 \mathrm{H}), 2.38 \sim 2.32(\mathrm{~m}, 2 \mathrm{H}), 2.09 \sim 2.02(\mathrm{~m}, 2 \mathrm{H}), 2.00(\mathrm{~s}$, $3 \mathrm{H}), 1.70 \sim 1.68(\mathrm{~m}, 6 \mathrm{H}), 1.59(\mathrm{~s}, 1 \mathrm{H}) ;{ }^{13} \mathrm{C}$ NMR $(100$ $\left.\mathrm{MHz}, \mathrm{CDCl}_{3}\right) \delta: 189.7,170.3,166.8,162.8,135.2,132.2$, $120.9,52.5,52.3,49.2,39.7,29.8,28.5,25.9,19.9,18.0$; HRMS calcd for $\mathrm{C}_{16} \mathrm{H}_{22} \mathrm{O}_{5} \mathrm{Na}[\mathrm{M}+\mathrm{Na}]^{+} 317.1359$, found 317.1364 .

\subsection{3中间体 7 和 12 的合成}

将化合物 6 (11.2 g, $38.1 \mathrm{mmol}$ )溶于四氢呋喃(THF)
$(100 \mathrm{~mL})$ 中, 冷却至 $0{ }^{\circ} \mathrm{C}$, 分批加入 $\mathrm{NaH}(60 \%$ in mineral oil, $1.7 \mathrm{~g}, 41.9 \mathrm{mmol}$ ), 同时保持反应液温度在 $5{ }^{\circ} \mathrm{C}$ 以下. 在该温度下搅拌 $1 \mathrm{~h}$ 后, 滴加甲基锂 $\left(1.6 \mathrm{~mol} \cdot \mathrm{L}^{-1}\right.$ in $\mathrm{Et}_{2} \mathrm{O}, 47.6 \mathrm{~mL}, 76.2 \mathrm{mmol}$ ), 并将反应液在 $0{ }^{\circ} \mathrm{C}$ 下摚 拌 $3 \mathrm{~h}, \mathrm{TLC}$ 监测. 反应毕, 用饱和氯化铵溶液 $(12 \mathrm{~mL})$ 淬 灭反应, 分液, 减压浓缩有机相, 水相用乙酸乙酯萃取 $(40 \mathrm{~mL} \times 3)$, 合并有机相, 有机相用水洗, 饱和食盐水 洗, 无水硫酸钠干燥, 过滤减压浓缩, 粗产物无需进一 步纯化, 直接投下一步.

将粗产物溶于 THF $(100 \mathrm{~mL})$ 中, 冷却至 $0{ }^{\circ} \mathrm{C}$, 分批 加入 $\mathrm{NaH}(60 \%$ in mineral oil, $1.7 \mathrm{~g}, 41.9 \mathrm{mmol})$, 在该温 度下搅拌 $1 \mathrm{~h}$ 后, 加入相应的溴代物 $(57.2 \mathrm{mmol})$. 随后 将反应液升至室温, 搅拌过夜, TLC 监测. 反应毕, 用饱 和氯化铵溶液 ( $30 \mathrm{~mL}$ ) 淬灭反应, 分液, 减压浓缩有机 相, 水相用乙酸乙酯萃取 $(50 \mathrm{~mL} \times 3)$, 合并有机相, 有 机相用水洗, 饱和食盐水洗, 无水硫酸钠干燥, 过滤减 压浓缩, 并进行柱层析纯化 [洗脱剂: $V$ (石油醚) : $V$ (乙 酸乙酯) $=10: 1$, 得到中间体 7 和 12 .

（土 )-3-乙酰基-4-甲基-1,5-双(3-甲基丁-2-烯-1基)-2-氧亚基环己基-3-烯-1-羧酸甲酯(7): 黄色油状物, $8.3 \mathrm{~g}$, 产率 $63 \%$. ${ }^{1} \mathrm{H}$ NMR (400 MHz, $\left.\mathrm{CDCl}_{3}\right) \delta: 5.08 \sim$ $5.01(\mathrm{~m}, 2 \mathrm{H}), 3.72(\mathrm{~s}, 3 \mathrm{H}), 2.63 \sim 2.56(\mathrm{~m}, 1 \mathrm{H}), 2.49 \sim$ $2.34(\mathrm{~m}, 3 \mathrm{H}), 2.29(\mathrm{~s}, 3 \mathrm{H}), 2.27 \sim 2.21(\mathrm{~m}, 1 \mathrm{H}), 2.11 \sim$ $2.05(\mathrm{~m}, 2 \mathrm{H}), 1.92(\mathrm{~s}, 3 \mathrm{H}), 1.71(\mathrm{~s}, 3 \mathrm{H}), 1.68(\mathrm{~s}, 3 \mathrm{H}), 1.61$ $(\mathrm{s}, 6 \mathrm{H}) ;{ }^{13} \mathrm{C}$ NMR $\left(100 \mathrm{MHz}, \mathrm{CDCl}_{3}\right) \delta: 204.3,194.8$, 173.0, 160.6, 139.1, 135.5, 134.9, 120.6, 118.8, 56.6, 52.6, 38.6, 33.0, 31.9, 31.4, 30.5, 26.1, 26.0, 19.6, 18.2, 18.1; HRMS calcd for $\mathrm{C}_{21} \mathrm{H}_{30} \mathrm{O}_{4} \mathrm{Na}[\mathrm{M}+\mathrm{Na}]^{+} 369.2036$, found 369.2041 .

(土)-3-乙酰基-1-烯丙基-4-甲基-5-(3-甲基丁-2-烯1-基)-2-氧亚基环己基-3-烯-1-羧酸甲酯(12): 淡黄色油 状物, $8.0 \mathrm{~g}$, 产率 66\%. ${ }^{1} \mathrm{H}$ NMR $\left(400 \mathrm{MHz}, \mathrm{CDCl}_{3}\right) \delta$ : $5.81 \sim 5.71(\mathrm{~m}, 1 \mathrm{H}), 5.11 \sim 5.03(\mathrm{~m}, 3 \mathrm{H}), 3.70(\mathrm{~s}, 3 \mathrm{H})$, $2.67 \sim 2.61(\mathrm{~m}, 1 \mathrm{H}), 2.47 \sim 2.35(\mathrm{~m}, 3 \mathrm{H}), 2.26(\mathrm{~s}, 3 \mathrm{H})$, $2.24 \sim 2.22(\mathrm{~m}, 1 \mathrm{H}), 2.12 \sim 1.99(\mathrm{~m}, 2 \mathrm{H}), 1.91(\mathrm{~s}, 3 \mathrm{H})$, 1.69 (s, 3H), $1.58(\mathrm{~s}, 3 \mathrm{H}) ;{ }^{13} \mathrm{C}$ NMR $\left(100 \mathrm{MHz}, \mathrm{CDCl}_{3}\right) \delta$ : $204.1,194.5,172.5,160.6,139.0,135.0,133.5,120.4$, $119.2,56.3,52.6,38.0,37.5,33.0,31.5,30.5,25.9,19.5$, 18.1; HRMS calcd for $\mathrm{C}_{19} \mathrm{H}_{26} \mathrm{O}_{4} \mathrm{Na}[\mathrm{M}+\mathrm{Na}]^{+} 341.1723$, found 341.1727 .

\subsection{4中间体 8 和 $\mathbf{1 3}$ 的合成}

将干燥的 $\mathrm{LiCl}(1.7 \mathrm{~g}, 39.3 \mathrm{mmol}$ ) 和 $\mathrm{CuI}(7.3 \mathrm{~g}, 38.4$ $\mathrm{mmol})$ 溶于 THF $(70 \mathrm{~mL})$ 中, 室温搅拌 $5 \mathrm{~min}$. 随后将反 应液冷却至 $-78{ }^{\circ} \mathrm{C}$, 滴加 $\mathrm{MeMgBr}\left(3 \mathrm{~mol} \cdot \mathrm{L}^{-1}\right.$ in $\mathrm{Et}_{2} \mathrm{O}$, $12.8 \mathrm{~mL}, 38.4 \mathrm{mmol}), \mathrm{TMSCl}(4.9 \mathrm{~mL}, 38.4 \mathrm{mmol})$ 和溶于 
THF $(70 \mathrm{~mL})$ 的相应的中间体 $(19.2 \mathrm{mmol}),-78{ }^{\circ} \mathrm{C}$ 下搅 拌 $5 \mathrm{~h}, \mathrm{TLC}$ 监测. 反应毕, 加入饱和 $\mathrm{NH}_{4} \mathrm{Cl}: 2 \mathrm{~mol} / \mathrm{L}$ $\mathrm{HCl}(1 ： 1,350 \mathrm{~mL})$ 水解反应, 减压浓缩有机相, 水相用 乙酸乙酯萃取 $(300 \mathrm{~mL} \times 3)$, 合并有机相, 有机相用 $\mathrm{NH}_{4} \mathrm{Cl}: \mathrm{NH}_{3} \cdot \mathrm{H}_{2} \mathrm{O}(V: V=1: 1$, 直到有机层无色)洗, 饱和食盐水洗, 无水硫酸钠干燥, 过滤减压浓缩, 并进 行柱层析纯化 [洗脱剂: $V$ (石油醚)： $V$ (乙酸乙酯 $)=50$ : 1], 得到中间体 $\mathbf{8}$ 和 $\mathbf{1 3}$.

(土)-3-乙酰基-4,4-二甲基-1,5-双(3-甲基丁-2-烯-1基)-2-氧亚基环己基-1-甲酸甲酯(8): 淡黄色油状物, 6.6 $\mathrm{g}$, 产率 96\%. ${ }^{1} \mathrm{H}$ NMR $\left(400 \mathrm{MHz}, \mathrm{CDCl}_{3}\right) \delta: 5.10 \sim 5.04$ $(\mathrm{m}, 2 \mathrm{H}), 3.77(\mathrm{~s}, 3 \mathrm{H}), 3.53(\mathrm{~s}, 1 \mathrm{H}), 2.51 \sim 2.40(\mathrm{~m}, 2 \mathrm{H})$, $2.33 \sim 2.26(\mathrm{~m}, 2 \mathrm{H}), 2.09(\mathrm{~s}, 3 \mathrm{H}), 2.02 \sim 1.91(\mathrm{~m}, 2 \mathrm{H})$, $1.72(\mathrm{~s}, 12 \mathrm{H}), 1.30 \sim 1.20(\mathrm{~m}, 1 \mathrm{H}), 1.11(\mathrm{~s}, 6 \mathrm{H}) ;{ }^{13} \mathrm{C} \mathrm{NMR}$ $\left(100 \mathrm{MHz}, \mathrm{CDCl}_{3}\right) \delta: 205.2,204.1,172.3,135.7,133.0$, 122.8, 118.6, 69.8, 61.1, 52.5, 43.0, 40.8, 33.3, 32.7, 32.2, 27.0, 26.1, 26.0, 24.1, 18.2, 16.1; HRMS calcd for $\mathrm{C}_{22} \mathrm{H}_{34} \mathrm{O}_{4} \mathrm{Na}[\mathrm{M}+\mathrm{Na}]^{+}$385.2349, found 385.2353.

(土)-3-乙酰基-1-烯丙基-4,4-二甲基-5-(3-甲基丁-2烯-1-基)-2-氧亚基环己基-1-甲酸甲酯(13)：黄色油状物, $5.8 \mathrm{~g}$, 产率 $91 \% .{ }^{1} \mathrm{H}$ NMR $\left(400 \mathrm{MHz}, \mathrm{CDCl}_{3}\right) \delta: 5.78 \sim$ $5.70(\mathrm{~m}, 1 \mathrm{H}), 5.05 \sim 4.99(\mathrm{~m}, 3 \mathrm{H}), 3.73(\mathrm{~s}, 3 \mathrm{H}), 3.51(\mathrm{~s}$, $1 \mathrm{H}), 2.54 \sim 2.39(\mathrm{~m}, 2 \mathrm{H}), 2.31 \sim 2.23(\mathrm{~m}, 2 \mathrm{H}), 2.08(\mathrm{~s}$, $3 \mathrm{H}), 1.99 \sim 1.87(\mathrm{~m}, 2 \mathrm{H}), 1.61(\mathrm{~s}, 6 \mathrm{H}), 1.29 \sim 1.24(\mathrm{~m}$, $1 \mathrm{H}), 1.10(\mathrm{~s}, 3 \mathrm{H}), 1.02(\mathrm{~s}, 3 \mathrm{H}) ;{ }^{13} \mathrm{C} \mathrm{NMR}(100 \mathrm{MHz}$, $\left.\mathrm{CDCl}_{3}\right) \delta: 204.8,203.9,172.1,133.5,133.2,122.6,118.9$, $70.1,60.7,44.0,41.9,39.0,34.8,33.1,27.1,26.5,24.9$, 18.0, 16.2; HRMS calcd for $\mathrm{C}_{20} \mathrm{H}_{30} \mathrm{O}_{4} \mathrm{Na}[\mathrm{M}+\mathrm{Na}]^{+}$ 357.2036, found 357.2041.

3.2.5 (土)-3-乙酰基-4,4-二甲基-1,3,5-三(3-甲基丁2-烯-1-基)-2-氧亚基环已基-1-甲酸甲酯(9)的合成

将配体 $\mathrm{SIMES}^{*} \mathrm{PF}_{6}(248 \mathrm{mg}, 0.6 \mathrm{mmol})$ 溶于甲基叔 丁基醚(MTBE) $(11 \mathrm{~mL})$ 中, 并加入 $\mathrm{KO}^{t} \mathrm{Am}(25 \%$ in toluene, $276 \mathrm{mg}, 0.6 \mathrm{mmol})$, 在 $60{ }^{\circ} \mathrm{C}$ 下搅拌 $1 \mathrm{~h}$ 后, 冷却至 室温, 加入 $\mathrm{Bu}_{4} \mathrm{~N}\left[\mathrm{Fe}(\mathrm{CO})_{3}(\mathrm{NO})\right](247.2 \mathrm{mg}, 0.6 \mathrm{mmol})$, 并将反应液在 $60{ }^{\circ} \mathrm{C}$ 下继续搅拌 $1 \mathrm{~h}$ (反应液 1). 同时, 将中间体 6 (2.2 g, $6 \mathrm{mmol})$ 溶于 THF (11 mL)中, 冷却至 $0{ }^{\circ} \mathrm{C}$, 并加入 $\mathrm{LiH}(52.8 \mathrm{mg}, 6.6 \mathrm{mmol})$, 在该温度下搅拌 $30 \mathrm{~min}$, 升至室温搅拌 $30 \mathrm{~min}$ 后, 将此反应液和 2-甲基 -3-丁烯-2-碳酸异丁酯(2.2 g, $12 \mathrm{mmol})$ 滴加至反应液 1 中, $100{ }^{\circ} \mathrm{C}$ 下搅拌过夜, TLC 监测. 反应毕, 将反应液通 过二氧化硅垫过滤, 减压浓缩, 并进行柱层析纯化 $[$ 洗 脱剂: $V$ (石油醚) $: V($ 乙酸乙酯 $)=100 ： 1]$, 得到淡黄色 油状物 9 (1.1 g, 产率 44\%). ${ }^{1} \mathrm{H} \mathrm{NMR}\left(400 \mathrm{MHz}, \mathrm{CDCl}_{3}\right)$ $\delta: 5.16 \sim 5.11(\mathrm{~m}, 1 \mathrm{H}), 5.05 \sim 5.02(\mathrm{~m}, 1 \mathrm{H}), 4.66 \sim 4.61$ $(\mathrm{m}, 1 \mathrm{H}), 3.75(\mathrm{~s}, 3 \mathrm{H}), 3.06 \sim 2.99(\mathrm{~m}, 1 \mathrm{H}), 2.37 \sim 2.25(\mathrm{~m}$, $3 \mathrm{H}), 2.22 \sim 2.16(\mathrm{~m}, 1 \mathrm{H}), 2.12(\mathrm{~s}, 3 \mathrm{H}), 2.03 \sim 1.99(\mathrm{~m}$, $2 \mathrm{H}), 1.81 \sim 1.77(\mathrm{~m}, 2 \mathrm{H}), 1.70(\mathrm{~s}, 6 \mathrm{H}), 1.61 \sim 1.57(\mathrm{~m}$, 12H), 1.04 (s, 3H), 0.99 (s, 3H); ${ }^{13} \mathrm{C}$ NMR (100MHz, $\left.\mathrm{CDCl}_{3}\right) \delta: 210.1,206.9,173.3,135.5,133.2,132.9,133.0$, $119.7,119.3,73.1,61.0,52.6,40.8,38.3,33.4,33.0,31.6$, 31.3, 27.7, 26.1, 26.1, 22.2, 21.8, 18.4, 18.2, 18.0; HRMS calcd for $\mathrm{C}_{27} \mathrm{H}_{42} \mathrm{O}_{4} \mathrm{Na}[\mathrm{M}+\mathrm{Na}]^{+}$453.2975, found 453.2980 .

\subsection{6中间体 $8 \mathrm{a}, 8 \mathrm{~b}, 13 \mathrm{a}, 13 \mathrm{~b}$ 的合成}

将相应的环己酮中间体 (4.5 mmol)溶于 DMF (21 $\mathrm{mL})$, 冷却至 $0{ }^{\circ} \mathrm{C}$, 分批加入 $\mathrm{NaH}(60 \%$ in mineral oil, $216 \mathrm{mg}, 5.4 \mathrm{mmol})$, 在该温度下搅拌 $1 \mathrm{~h}$. 然后滴加氯甲 酸烯丙酯 $(0.56 \mathrm{~mL}, 5.4 \mathrm{mmol})$, 将反应液升至室温, 搅 拌过夜, TLC 监测. 反应毕, 用饱和氯化铵溶液 $(20 \mathrm{~mL})$ 淬灭反应，分液，水相用乙酸乙酯萃取 $(80 \mathrm{~mL} \times 3)$, 合 并有机相，有机相用水洗，饱和食盐水洗，无水硫酸钠 干燥，过滤减压浓缩，并进行柱层析纯化 [洗脱剂: $V($ 石 油醚)： $V$ (乙酸乙酯 $)=100 ： 1]$, 得到中间体 $\mathbf{8 a}, \mathbf{8 b}, \mathbf{1 3 a}$, $13 b$.

(土)-3-乙酰基-2-(((烯丙氧基)羰基)氧基)-4,4-二甲 基-1,5-双(3-甲基丁-2-烯-1-基)环已基-2-烯-1-羧酸酯 (8a): 黄色油状物, $640 \mathrm{mg}$, 产率 34\%. ${ }^{1} \mathrm{H}$ NMR (400 $\left.\mathrm{MHz}, \mathrm{CDCl}_{3}\right) \delta: 5.99 \sim 5.89(\mathrm{~m}, 1 \mathrm{H}), 5.41 \sim 5.29(\mathrm{~m}, 2 \mathrm{H})$, $4.98 \sim 4.96(\mathrm{~m}, 2 \mathrm{H}), 4.68 \sim 4.66(\mathrm{~m}, 2 \mathrm{H}), 3.69(\mathrm{~s}, 3 \mathrm{H})$, $2.61 \sim 2.46(\mathrm{~m}, 2 \mathrm{H}), 2.35 \sim 2.28(\mathrm{~m}, 1 \mathrm{H}), 2.11 \sim 2.06(\mathrm{~m}$, $1 \mathrm{H}), 1.96(\mathrm{~s}, 3 \mathrm{H}), 1.82 \sim 1.78(\mathrm{~m}, 1 \mathrm{H}), 1.68 \sim 1.67(\mathrm{~d}, J=$ $3.1 \mathrm{~Hz}, 6 \mathrm{H}), 1.65 \sim 1.63(\mathrm{~m}, 1 \mathrm{H}), 1.61(\mathrm{~s}, 3 \mathrm{H}), 1.57(\mathrm{~s}$, $3 \mathrm{H}), 1.24 \sim 1.22(\mathrm{~m}, 1 \mathrm{H}), 1.19(\mathrm{~s}, 3 \mathrm{H}), 1.13(\mathrm{~s}, 3 \mathrm{H}) ;{ }^{13} \mathrm{C}$ NMR (100 MHz, $\left.\mathrm{CDCl}_{3}\right) \delta: 202.9,172.1,152.0,150.1$, $135.7,135.5,133.2,131.2,123.0,119.6,118.4,69.2,60.6$, 52.6, 44.1, 41.9, 33.2, 32.3, 27.6, 26.5, 26.2, 26.1, 19.1, 18.5, 18.0, 18.0; HRMS calcd for $\mathrm{C}_{26} \mathrm{H}_{39} \mathrm{O}_{6}[\mathrm{M}+\mathrm{H}]^{+}$ 447.2741, found 447.2738.

(土)-3-(1-(((烯丙氧基)羰基)氧基)亚乙基)-4,4-二甲 基-1,5-双(3-甲基丁-2-烯-1-基)-2-氧亚基环己烷-1-羧酸 酯 $(8 \mathrm{~b})$ : 黄色油状物, $960 \mathrm{mg}$, 产率 50\%. ${ }^{1} \mathrm{H}$ NMR (400 $\left.\mathrm{MHz}, \mathrm{CDCl}_{3}\right) \delta: 5.93 \sim 5.83(\mathrm{~m}, 1 \mathrm{H}), 5.36 \sim 5.25(\mathrm{~m}, 2 \mathrm{H})$, $5.01 \sim 4.94(\mathrm{~m}, 2 \mathrm{H}), 4.61 \sim 4.53(\mathrm{~m}, 2 \mathrm{H}), 3.63(\mathrm{~s}, 3 \mathrm{H})$, $2.79 \sim 2.74(\mathrm{~m}, 1 \mathrm{H}), 2.40 \sim 2.34(\mathrm{~m}, 1 \mathrm{H}), 2.27(\mathrm{~s}, 3 \mathrm{H})$, $2.15 \sim 2.10(\mathrm{~m}, 1 \mathrm{H}), 2.04 \sim 1.97(\mathrm{~m}, 1 \mathrm{H}), 1.83 \sim 1.79(\mathrm{~m}$, $1 \mathrm{H}), 1.74 \sim 1.70(\mathrm{~m}, 1 \mathrm{H}), 1.68(\mathrm{~s}, 6 \mathrm{H}), 1.61(\mathrm{~s}, 3 \mathrm{H}), 1.58$ $(\mathrm{s}, 3 \mathrm{H}), 1.47 \sim 1.40(\mathrm{~m}, 1 \mathrm{H}), 1.15(\mathrm{~s}, 3 \mathrm{H}), 1.06(\mathrm{~s}, 3 \mathrm{H})$; ${ }^{13} \mathrm{C}$ NMR $\left(100 \mathrm{MHz}, \mathrm{CDCl}_{3}\right) \delta: 204.1,173.3,152.5,144.2$, $140.6,135.1,133.1,131.2,123.1,119.5,119.4,69.3,52.7$, 
$50.5,41.0,38.1,34.0,32.4,30.4,27.8,26.2,26.0,25.3$, 21.2, 18.2, 18.0; HRMS calcd for $\mathrm{C}_{26} \mathrm{H}_{39} \mathrm{O}_{6}[\mathrm{M}+\mathrm{H}]^{+}$ 447.2471, found 447.2736.

(土)-3-乙酰基-1-烯丙基-2-(( (烯丙氧基)羰基)氧 基)-4,4-二甲基-5-(3-甲基丁-2-烯-1-基)环已基-2-烯-1-羧 酸酯(13a): 黄色油状物, $567 \mathrm{mg}$, 产率 $32 \% .{ }^{1} \mathrm{H} \mathrm{NMR}$ $\left(400 \mathrm{MHz}, \mathrm{CDCl}_{3}\right) \delta: 5.99 \sim 5.89(\mathrm{~m}, 1 \mathrm{H}), 5.72 \sim 5.62(\mathrm{~m}$, $1 \mathrm{H}), 5.41 \sim 5.28(\mathrm{~m}, 2 \mathrm{H}), 5.09 \sim 4.99(\mathrm{~m}, 3 \mathrm{H}), 4.68 \sim 4.66$ $(\mathrm{m}, 2 \mathrm{H}), 3.69(\mathrm{~s}, 3 \mathrm{H}), 2.70 \sim 2.65(\mathrm{~m}, 1 \mathrm{H}), 2.47 \sim 2.42(\mathrm{~m}$, $1 \mathrm{H}), 2.34 \sim 2.27(\mathrm{~m}, 1 \mathrm{H}), 2.11 \sim 2.07(\mathrm{~m}, 1 \mathrm{H}), 1.96(\mathrm{~s}$, $3 \mathrm{H}), 1.90 \sim 1.85(\mathrm{M}, 1 \mathrm{H}), 1.73 \sim 1.70(\mathrm{~m}, 1 \mathrm{H}), 1.67(\mathrm{~s}$, $3 \mathrm{H}), 1.57(\mathrm{~s}, 3 \mathrm{H}), 1.25 \sim 1.20(\mathrm{~m}, 1 \mathrm{H}), 1.18(\mathrm{~s}, 3 \mathrm{H}), 1.15$ (s, 3H); ${ }^{13} \mathrm{C}$ NMR $\left(100 \mathrm{MHz}, \mathrm{CDCl}_{3}\right) \delta: 202.5,171.7$, $151.9,150.5,135.4,133.3,133.0,131.2,122.9,119.6$, $119.4,69.2,60.0,52.6,44.0,41.8,39.1,31.9,27.5,26.6$, 25.9, 19.1, 18.5, 18.0; HRMS calcd for $\mathrm{C}_{24} \mathrm{H}_{34} \mathrm{O}_{6} \mathrm{Na}[\mathrm{M}+$ $\mathrm{Na}]^{+}$441.2248, found 441.2251 .

(土)-1-烯丙基-3-(1-((( 烯丙氧基)羰基)氧基)亚乙 基)-4,4-二甲基-5-(3-甲基丁-2-烯-1-基)-2-氧亚基环己烷1-羧酸酯 $(13 \mathrm{~b})$ : 黄色油状物, $1.1 \mathrm{~g}$, 产率 $63 \%$. ${ }^{1} \mathrm{H}$ NMR $\left(400 \mathrm{MHz}, \mathrm{CDCl}_{3}\right) \delta: 5.93 \sim 5.84(\mathrm{~m}, 1 \mathrm{H}), 5.71 \sim 5.61(\mathrm{~m}$, $1 \mathrm{H}), 5.37 \sim 5.26(\mathrm{~m}, 2 \mathrm{H}), 5.10 \sim 5.02(\mathrm{~m}, 3 \mathrm{H}), 4.58 \sim 4.56$ $(\mathrm{m}, 2 \mathrm{H}), 3.63(\mathrm{~s}, 3 \mathrm{H}), 2.91 \sim 2.86(\mathrm{~m}, 1 \mathrm{H}), 2.38 \sim 2.35(\mathrm{~m}$, $1 \mathrm{H}), 2.27(\mathrm{~s}, 3 \mathrm{H}), 2.16 \sim 2.11(\mathrm{~m}, 1 \mathrm{H}), 2.06 \sim 1.97(\mathrm{~m}$, $1 \mathrm{H}), 1.90 \sim 1.86(\mathrm{~m}, 1 \mathrm{H}), 1.67(\mathrm{~s}, 3 \mathrm{H}), 1.59(\mathrm{~s}, 3 \mathrm{H}), 1.50 \sim$ $1.43(\mathrm{~m}, 1 \mathrm{H}), 1.27 \sim 1.22(\mathrm{~m}, 1 \mathrm{H}), 1.16(\mathrm{~s}, 3 \mathrm{H}), 1.08(\mathrm{~s}$, $3 \mathrm{H}) ;{ }^{13} \mathrm{C}$ NMR (100 MHz, $\left.\mathrm{CDCl}_{3}\right) \delta: 203.9,172.9,152.5$, $144.0,140.8,133.5,133.3,131.1,123.1,119.6,119.3$, 69.4, 52.7, 50.2, 40.8, 39.7, 38.1, 32.4, 29.9, 27.6, 25.9, 25.2, 21.2, 18.0; HRMS calcd for $\mathrm{C}_{24} \mathrm{H}_{34} \mathrm{O}_{6} \mathrm{Na}[\mathrm{M}+\mathrm{Na}]^{+}$ 441.2248 , found 441.2253 .

\subsection{7中间体 14 和 17 的合成}

将三(二亚苄基丙酮)二钯(0)-氯仿加合物(201 mg, $0.2 \mathrm{mmol}$ )和三(对甲苯基)膦 $(293 \mathrm{mg}, 1.0 \mathrm{mmol}$ )溶于甲 苯 $(11 \mathrm{~mL})$ 中, 室温搅拌 $1 \mathrm{~h}$. 随后滴加溶于甲苯的相应 的中间体 (4 mmol), 室温摚拌过夜, TLC 监测. 反应毕, 将反应液通过二氧化硅垫过滤, 减压浓缩, 并进行柱层 析纯化 [洗脱剂: $V$ (石油醚) $: V$ (乙酸乙酯 $)=100 ： 1$ ], 得 到中间体 14 和 17.

(土)-3-乙酰基-1,3-二烯丙基-4,4-二甲基-5-(3-甲基 丁-2-烯-1-基)-2-氧亚基环己烷-1-甲酸甲酯(14): 淡黄色 油状物, $1.1 \mathrm{~g}$, 产率 73\%. ${ }^{1} \mathrm{H}$ NMR (400 MHz, $\left.\mathrm{CDCl}_{3}\right) \delta$ : $5.89 \sim 5.79(\mathrm{~m}, 1 \mathrm{H}), 5.37 \sim 5.27(\mathrm{~m}, 1 \mathrm{H}), 5.17 \sim 5.15(\mathrm{~m}$, $1 \mathrm{H}), 5.10 \sim 5.05(\mathrm{~m}, 2 \mathrm{H}), 4.94 \sim 4.88(\mathrm{~m}, 2 \mathrm{H}), 3.76(\mathrm{~s}$, $3 \mathrm{H}), 3.19 \sim 3.14(\mathrm{~m}, 1 \mathrm{H}), 2.68 \sim 2.63(\mathrm{~m}, 1 \mathrm{H}), 2.30 \sim 2.18$ (m, 3H), $2.13(\mathrm{~s}, 3 \mathrm{H}), 2.07 \sim 2.05(\mathrm{~m}, 2 \mathrm{H}), 1.81 \sim 1.76(\mathrm{~m}$, 2H), $1.70(\mathrm{~s}, 3 \mathrm{H}), 1.61(\mathrm{~s}, 3 \mathrm{H}) ;{ }^{13} \mathrm{C}$ NMR $(100 \mathrm{MHz}$, $\left.\mathrm{CDCl}_{3}\right) \delta: 209.1,206.3,172.9,134.0,133.7,133.5,122.9$, 199.8, 117.6, 73,7, 59.8, 52.6, 40.6, 38.9, 37.7, 37.4, 32.9, $31.1,27.4,25.9,22.3,21.9,18.1$; HRMS calcd for $\mathrm{C}_{23} \mathrm{H}_{34} \mathrm{O}_{4} \mathrm{Na}[\mathrm{M}+\mathrm{Na}]^{+}$397.2349, found 397.2354.

(土)-3-乙酰基-3-烯丙基-4,4-二甲基-1,5-双(3-甲基 丁-2-烯-1-基)-2-氧亚基环已烷-1-甲酸甲酯(17)：淡黄色 油状物, $1.2 \mathrm{~g}$, 产率 $75 \%$. ${ }^{1} \mathrm{H}$ NMR $\left(400 \mathrm{MHz}, \mathrm{CDCl}_{3}\right) \delta$ : $5.38 \sim 5.27(\mathrm{~m}, 1 \mathrm{H}), 5.17 \sim 5.14(\mathrm{~m}, 1 \mathrm{H}), 5.02 \sim 5.00(\mathrm{~m}$, $1 \mathrm{H}), 4.94 \sim 4.87(\mathrm{~m}, 2 \mathrm{H}), 3.75(\mathrm{~s}, 3 \mathrm{H}), 3.20 \sim 3.15(\mathrm{~m}$, $1 \mathrm{H}), 2.46 \sim 2.36(\mathrm{~m}, 2 \mathrm{H}), 2.27 \sim 2.17(\mathrm{~m}, 2 \mathrm{H}), 2.11(\mathrm{~s}$, $3 \mathrm{H}), 2.02 \sim 2.00(\mathrm{~m}, 2 \mathrm{H}), 1.78 \sim 1.74(\mathrm{~m}, 1 \mathrm{H}), 1.71(\mathrm{~s}$, $3 \mathrm{H}), 1.70(\mathrm{~s}, 3 \mathrm{H}), 1.63(\mathrm{~s}, 3 \mathrm{H}), 1.59$ (s, 3H), $1.27 \sim 1.14$ (m, 1H), 1.06 (s, 3H), 0.98 (s, 3H); ${ }^{13} \mathrm{C}$ NMR $(100 \mathrm{MHz}$, $\left.\mathrm{CDCl}_{3}\right) \delta: 209.4,206.5,173.2,135.7,134.1,133.3,122.9$, 119.6, 117.5, 73.6, 60.3, 52.6, 40.7, 38.2, 37.5, 33.1, 32.9, 31.3, 27.6, 26.1, 26.1, 22.3, 21.8, 18.4, 18.0; HRMS calcd for $\mathrm{C}_{25} \mathrm{H}_{38} \mathrm{O}_{4} \mathrm{Na}[\mathrm{M}+\mathrm{Na}]^{+}$425.2662, found 425.2658.

3.2.8中间体 $10,15,18$ 的合成

将相应的中间体 $(3.0 \mathrm{mmol})$ 溶于 THF $(90 \mathrm{~mL})$ 中, 冷却至 $0{ }^{\circ} \mathrm{C}$, 加入叔丁醇钾 $(680 \mathrm{mg}, 6 \mathrm{mmol})$, 在该温 度下搅拌 $45 \mathrm{~min}$, 随后升至室温, 用饱和氯化铵溶液淬 灭反应, 减压浓缩有机相, 水相用乙酸乙酯萃取 (50 $\mathrm{mL} \times 3)$, 合并有机相, 有机相用水洗, 饱和食盐水洗, 无水硫酸钠干燥, 过滤减压浓缩, 并进行柱层析纯化 [洗脱剂: $V$ (二氯甲烷 $): V($ 甲醇 $)=100 ： 1$ ] , 得到中间体 10,15 和 18.

(土)-5,5-二甲基-4,6,8-三(3-甲基丁-2-烯-1-基)双环 [3.3.1]壬烷-1,3,9-三酮(10): 白色固体，956 mg, 产率 $80 \%$. m.p. $116 \sim 119{ }^{\circ} \mathrm{C} ;{ }^{1} \mathrm{H}$ NMR (300 MHz, $\mathrm{CDCl}_{3}$ ) $\delta$ : $5.12 \sim 5.08(\mathrm{~m}, 1 \mathrm{H}), 4.94 \sim 4.83(\mathrm{~m}, 2 \mathrm{H}), 3.56(\mathrm{~d}, J=17.2$ $\mathrm{Hz}, 1 \mathrm{H}), 2.95(\mathrm{~d}, J=17.2 \mathrm{~Hz}, 1 \mathrm{H}), 2.56 \sim 2.54(\mathrm{~m}, 2 \mathrm{H})$, $2.49 \sim 2.47(\mathrm{~m}, 2 \mathrm{H}), 2.19 \sim 2.14(\mathrm{~m}, 2 \mathrm{H}), 2.07 \sim 2.00(\mathrm{~m}$, $1 \mathrm{H}), 1.66 \sim 1.65(\mathrm{~m}, 9 \mathrm{H}), 1.61(\mathrm{~s}, 6 \mathrm{H}), 1.51(\mathrm{~s}, 3 \mathrm{H}), 1.48 \sim$ $1.39(\mathrm{~m}, 1 \mathrm{H}), 1.33 \sim 1.27(\mathrm{~m}, 1 \mathrm{H}), 1.25(\mathrm{~s}, 3 \mathrm{H}), 0.96(\mathrm{~s}$, $3 \mathrm{H}) ;{ }^{13} \mathrm{C}$ NMR (75 MHz, $\mathrm{CDCl}_{3}$ ) $\delta: 210.6,203.0,202.8$, 136.6, 135.7, 133.8, 122.7, 118.2, 117.6, 70.3, 64.9, 62.6, 51.3, 46.5, 40.6, 31.3, 29.3, 27.3, 26.5, 26.2, 26.2, 26.0, 23.0, 18.1, 18.0, 18.0; HRMS calcd for $\mathrm{C}_{26} \mathrm{H}_{38} \mathrm{O}_{3} \mathrm{Na}$ $421.2713[\mathrm{M}+\mathrm{Na}]^{+}$, found 421.2719 .

(土)-5,5-二甲基-4,8-二烯丙基-6-(3-甲基丁-2-烯-1基)双环[3.3.1]壬烷-1,3,9-三酮(15)：白色固体，832 mg, 产率 81\%. m.p. $128 \sim 131{ }^{\circ} \mathrm{C} ;{ }^{1} \mathrm{H}$ NMR $(400 \mathrm{MHz}$, $\left.\mathrm{CDCl}_{3}\right) \delta: 5.85 \sim 5.75(\mathrm{~m}, 1 \mathrm{H}), 5.69 \sim 5.59(\mathrm{~m}, 1 \mathrm{H})$, 
$5.10 \sim 5.01(\mathrm{~m}, 4 \mathrm{H}), 4.86 \sim 4.83(\mathrm{~m}, 1 \mathrm{H}), 3.61(\mathrm{~d}, J=17.3$ $\mathrm{Hz}, 1 \mathrm{H}), 2.89$ (d, J=17.2 Hz, 1H), 2.76 2.72 (m, 1H), $2.60 \sim 2.55(\mathrm{~m}, 1 \mathrm{H}), 2.48 \sim 2.42(\mathrm{~m}, 2 \mathrm{H}), 2.24 \sim 2.15(\mathrm{~m}$, $2 \mathrm{H}), 2.09 \sim 2.04(\mathrm{~m}, 1 \mathrm{H}), 1.66(\mathrm{~s}, 3 \mathrm{H}), 1.51(\mathrm{~s}, 3 \mathrm{H}), 1.48 \sim$ $1.40(\mathrm{~m}, 1 \mathrm{H}), 1.37 \sim 1.32(\mathrm{~m}, 1 \mathrm{H}), 1.24(\mathrm{~s}, 3 \mathrm{H}), 0.97(\mathrm{~s}$, $3 \mathrm{H}) ;{ }^{13} \mathrm{C}$ NMR (100 MHz, $\left.\mathrm{CDCl}_{3}\right) \delta: 210.0,202.4,202.3$, $134.0,132.5,132.2,122.5,120.7,119.9,71.0,64.7,63.3$, 51.5, 46.5, 41.0, 36.9, 32.6, 29.3, 26.4, 25.9, 23.1, 18.0; HRMS calcd for $\mathrm{C}_{22} \mathrm{H}_{30} \mathrm{O}_{3} \mathrm{Na}[\mathrm{M}+\mathrm{Na}]^{+}$365.2087, found 365.2092.

（土)-5,5-二甲基-4-烯丙基-6,8-二(3-甲基丁-2-烯-1基)双环[3.3.1]壬烷-1,3,9-三酮(18)：白色固体，801 mg， 产率 72\%. m.p. $124 \sim 126{ }^{\circ} \mathrm{C}$; ${ }^{1} \mathrm{H}$ NMR $(400 \mathrm{MHz}$, $\left.\mathrm{CDCl}_{3}\right) \delta: 5.72 \sim 5.60(\mathrm{~m}, 1 \mathrm{H}), 5.13 \sim 5.01(\mathrm{~m}, 3 \mathrm{H})$, $4.90 \sim 4.85(\mathrm{~m}, 1 \mathrm{H}), 3.74 \sim 3.56(\mathrm{~m}, 1 \mathrm{H}), 2.92 \sim 2.88(\mathrm{~m}$, $1 \mathrm{H}), 2.77 \sim 2.72(\mathrm{~m}, 1 \mathrm{H}), 2.47 \sim 2.41(\mathrm{~m}, 2 \mathrm{H}), 2.22 \sim 2.15$ $(\mathrm{m}, 2 \mathrm{H}), 2.11 \sim 2.02(\mathrm{~m}, 2 \mathrm{H}), 1.69 \sim 1.63(\mathrm{~m}, 9 \mathrm{H}), 1.51(\mathrm{~s}$, $3 \mathrm{H}), 1.35 \sim 1.30(\mathrm{~m}, 1 \mathrm{H}), 1.23(\mathrm{~s}, 3 \mathrm{H}), 1.10 \sim 1.08(\mathrm{~m}$, $1 \mathrm{H}), 0.96(\mathrm{~s}, 3 \mathrm{H}) ;{ }^{13} \mathrm{C}$ NMR $\left(100 \mathrm{MHz}, \mathrm{CDCl}_{3}\right) \delta: 210.2$, $202.7,202.5,135.8,133.8,132.3,122.6,120.6,118.0$, $70.9,64.8,63.2,51.5,46.6,40.9,32.7,31.5,29.3,26.4$, 26.1, 25.9, 23.1, 18.1, 18.0; HRMS calcd for $\mathrm{C}_{24} \mathrm{H}_{34} \mathrm{O}_{3} \mathrm{Na}$ $[\mathrm{M}+\mathrm{Na}]^{+}$393.2400, found 393.2399.

3.2.9中间体 11, 16, 19 的合成

取相应的中间体 $(1.1 \mathrm{mmol})$ 溶于无水 THF 中, 在氮 气保护下缓慢加入三乙胺 $(0.4 \mathrm{~mL}, 3.3 \mathrm{mmol})$ 和溶于 THF 的 3,4-二乙酰氧基苯甲酰氧 $(815 \mathrm{mg}, 3.3 \mathrm{mmol})$, 搅 拌过夜. 反应毕, 加入饱和的氯化铵溶液, 减压浓缩有 机相，水相用乙酸乙酯萃取，合并有机相，有机相用水 洗, 饱和食盐水洗, 无水硫酸钠干燥, 减压浓缩, 粗产 物经过柱层析分离纯化 [洗脱剂： $V$ (二氯甲烷)： $V($ 甲 醇 $)=400 ： 1]$, 得到中间体 11, 16, 19.

（土 )-4-(5,5-二甲基-4,6,8-三(3-甲基丁-2-烯-1基)-1,3,9-三氧亚基双环[3.3.1]壬烷-2-羰基)-1,2-苯二乙 酸酯(11): 黄色油状物, $517 \mathrm{mg}$, 产率 76\%. ${ }^{1} \mathrm{H}$ NMR (400 $\left.\mathrm{MHz} \mathrm{CDCl}_{3}\right) \delta: 7.46 \sim 7.44(\mathrm{~m}, 1 \mathrm{H}), 7.21 \sim 7.19(\mathrm{~m}, 2 \mathrm{H})$, $4.85 \sim 4.83(\mathrm{~m}, 2 \mathrm{H}), 4.79 \sim 4.76(\mathrm{~m}, 1 \mathrm{H}), 2.29(\mathrm{~s}, 6 \mathrm{H})$, $2.24 \sim 2.17(\mathrm{~m}, 2 \mathrm{H}), 2.15 \sim 2.10(\mathrm{~m}, 2 \mathrm{H}), 2.04 \sim 1.97(\mathrm{~m}$, $2 \mathrm{H}), 1.96 \sim 1.81(\mathrm{~m}, 2 \mathrm{H}), 1.74(\mathrm{~s}, 6 \mathrm{H}), 1.70(\mathrm{~s}, 3 \mathrm{H}), 1.66$ $(\mathrm{s}, 3 \mathrm{H}), 1.58(\mathrm{~s}, 3 \mathrm{H}), 145 \sim 1.44(\mathrm{~m}, 1 \mathrm{H}), 1.42(\mathrm{~s}, 3 \mathrm{H})$, $1.13(\mathrm{~s}, 3 \mathrm{H}), 0.97(\mathrm{~s}, 3 \mathrm{H}) ;{ }^{13} \mathrm{C} \mathrm{NMR}\left(100 \mathrm{MHz}, \mathrm{CDCl}_{3}\right) \delta$ : 208.0, 197.4, 194.6, 193.6, 167.9, 167.6, 145.7, 141.6, $135.0,134.8,133.1,128.0,124.1,123.5,122.8,119.8$, $118.5,115.6,68.8,65.6,63.3,58.5,53.6,48.5,46.4,39.4$, 30.7, 29.0, 26.6, 26.2, 25.9, 22.6, 20.7, 18.2, 17.9; HRMS calcd for $\mathrm{C}_{37} \mathrm{H}_{46} \mathrm{O}_{8} \mathrm{Na}[\mathrm{M}+\mathrm{Na}]^{+}$641.3085, found 641.3083 .

(土)-4-(5,5-二甲基-4,8-二烯丙基-6-(3-甲基丁-2-烯 -1-基)-1,3,9-三氧亚基双环[3.3.1]壬烷-2-羰基)-1,2-苯二 乙酸酯(16): 黄色油状物, $482 \mathrm{mg}$, 产率 $78 \%$. ${ }^{1} \mathrm{H} \mathrm{NMR}$ $\left(400 \mathrm{MHz}, \mathrm{CDCl}_{3}\right) \delta: 7.41 \sim 7.40(\mathrm{~m}, 1 \mathrm{H}), 7.27 \sim 7.21(\mathrm{~m}$, 2H), $5.62 \sim 5.49(\mathrm{~m}, 1 \mathrm{H}), 5.12 \sim 5.05(\mathrm{~m}, 4 \mathrm{H}), 4.85 \sim 4.80$ $(\mathrm{m}, 2 \mathrm{H}), 2.63 \sim 2.60(\mathrm{~m}, 2 \mathrm{H}), 2.55 \sim 2.54(\mathrm{~m}, 2 \mathrm{H}), 2.29(\mathrm{~s}$, $6 \mathrm{H}), 1.99 \sim 1.96(\mathrm{~m}, 2 \mathrm{H}), 1.95 \sim 1.85(\mathrm{~m}, 2 \mathrm{H}), 1.61(\mathrm{~s}$, $3 \mathrm{H}), 1.46 \sim 1.44(\mathrm{~m}, 1 \mathrm{H}), 1.41(\mathrm{~s}, 3 \mathrm{H}), 1.12(\mathrm{~s}, 3 \mathrm{H}), 0.98$ $(\mathrm{s}, 3 \mathrm{H}) ;{ }^{13} \mathrm{C}$ NMR $\left(100 \mathrm{MHz}, \mathrm{CDCl}_{3}\right) \delta: 207.6,196.2$, $194.8,192.9,167.6,145.9,141.4,134.8,134.2,133.3$, $132.9,128.0,125.0,123.4,122.9,119.7,119.2,116.0$, 69.2, 65.7, 63.0, 58.1, 48.7, 46.5, 39.4, 36.0, 31.2, 29.0, 27.0, 25.8, 22.5, 20.7, 17.9; HRMS calcd for $\mathrm{C}_{33} \mathrm{H}_{38} \mathrm{O}_{8} \mathrm{Na}$ $[\mathrm{M}+\mathrm{Na}]^{+}$585.2459, found 585.2455.

(土)-4-(5,5-二甲基-4-烯丙基-6,8-二(3-甲基丁-2-烯1-基)-1,3,9-三氧亚基双环[3.3.1]壬烷-2-羰基)-1,2-苯二 乙酸酯(19): 黄色油状物, $487 \mathrm{mg}$, 产率 $75 \%$. ${ }^{1} \mathrm{H} \mathrm{NMR}$ $\left(500 \mathrm{MHz}, \mathrm{CDCl}_{3}\right) \delta: 7.60 \sim 7.59(\mathrm{~m}, 1 \mathrm{H}), 7.24 \sim 7.20(\mathrm{~m}$, $2 \mathrm{H}), 5.62 \sim 5.54(\mathrm{~m}, 1 \mathrm{H}), 5.08 \sim 5.00(\mathrm{~m}, 2 \mathrm{H}), 4.87 \sim 4.82$ $(\mathrm{m}, 2 \mathrm{H}), 2.61 \sim 2.54(\mathrm{~m}, 2 \mathrm{H}), 2.52 \sim 2.39(\mathrm{~m}, 2 \mathrm{H}), 2.29(\mathrm{~s}$, $6 \mathrm{H}), 2.04 \sim 1.96(\mathrm{~m}, 2 \mathrm{H}), 1.93 \sim 1.84(\mathrm{~m}, 2 \mathrm{H}), 1.74(\mathrm{~s}$, $3 \mathrm{H}), 1.70(\mathrm{~s}, 3 \mathrm{H}), 1.62(\mathrm{~s}, 3 \mathrm{H}), 1.42(\mathrm{~s}, 3 \mathrm{H}), 1.26 \sim 1.25$ $(\mathrm{m}, 1 \mathrm{H}), 1.12(\mathrm{~s}, 3 \mathrm{H}), 0.98(\mathrm{~s}, 3 \mathrm{H}) ;{ }^{13} \mathrm{C}$ NMR $(125 \mathrm{MHz}$, $\left.\mathrm{CDCl}_{3}\right) \delta: 207.8,196.4,194.6,193.1,167.9,167.6,145.8$, $141.4,135.1,134.8,133.2,128.13,124.55,123.46,122.84$, $119.57,116.16,115.88,69.0,65.6,63.2,58.3,48.7,46.5$, 39.4, 31.3, 30.8, 29.0, 26.2, 25.9, 22.5, 20.8, 20.6, 18.2, 17.9; HRMS calcd for $\mathrm{C}_{35} \mathrm{H}_{42} \mathrm{O}_{8} \mathrm{Na}[\mathrm{M}+\mathrm{Na}]^{+}$613.2772, found 613.2769 .

\section{2 .10 山竹醇新类似物的合成}

取相应的中间体 $\left(0.9 \mathrm{mmol}\right.$ )和 $\mathrm{K}_{2} \mathrm{CO}_{3}$ (496 mg, 3.6 $\mathrm{mmol})$ 于烧瓶中，抽空换氮，随后加入甲醇 $(15 \mathrm{~mL})$, 室 温搅拌 $1 \mathrm{~h}$. 反应毕, 加入饱和的氯化铵溶液, 用乙酸乙 酯萃取三次,合并有机相，有机相用饱和食盐水洗，无水 硫酸钠干燥, 减压浓缩, 粗产物柱层析分离纯化 [洗脱 剂: $V$ (二氯甲烷) $: V$ (甲醇 $)=50 ： 1]$, 得到最终产物.

8-异戊烯基(代)山竹醇(1)：黄色粘稠物， $342.2 \mathrm{mg}$, 产率 64\%. ${ }^{1} \mathrm{H}$ NMR (400 MHz, $\left.\mathrm{CD}_{3} \mathrm{OD}\right) \delta: 7.18 \sim 7.17(\mathrm{~m}$, $1 \mathrm{H}), 6.98 \sim 6.96(\mathrm{~m}, 1 \mathrm{H}), 6.71 \sim 6.69(\mathrm{~m}, 1 \mathrm{H}), 5.17 \sim 5.15$ $(\mathrm{m}, 1 \mathrm{H}), 4.94(\mathrm{~m}, 2 \mathrm{H}), 2.75 \sim 2.70(\mathrm{~m}, 1 \mathrm{H}), 2.59 \sim 2.49$ $(\mathrm{m}, 3 \mathrm{H}), 2.18 \sim 2.00(\mathrm{~m}, 4 \mathrm{H}), 1.72(\mathrm{~s}, 3 \mathrm{H}), 1.68(\mathrm{~s}, 6 \mathrm{H})$, $1.65(\mathrm{~s}, 6 \mathrm{H}), 1.48(\mathrm{~s}, 3 \mathrm{H}), 1.34 \sim 1.29(\mathrm{~m}, 1 \mathrm{H}), 1.24(\mathrm{~s}$, $3 \mathrm{H}), 1.01(\mathrm{~s}, 3 \mathrm{H}) ;{ }^{13} \mathrm{C} \mathrm{NMR}\left(100 \mathrm{MHz}, \mathrm{CD}_{3} \mathrm{OD}\right) \delta: 209.9$, 
$195.9,152.5,146.2,135.6,135.5,133.7,129.5,125.4$, $125.1,120.8,117.8,117.4,115.1,47.8,32.1,30.1,27.3$, 27.1, 26.3, 26.0, 23.2, 18.3, 18.2, 18.1; HRMS calcd for $\mathrm{C}_{33} \mathrm{H}_{42} \mathrm{O}_{6} \mathrm{Na}[\mathrm{M}+\mathrm{Na}]^{+}$557.2874, found 557.2871.

4,8-二烯丙基(代)山竹醇(2): 黄色粘稠物, $297.2 \mathrm{mg}$, 产率 $69 \% .{ }^{1} \mathrm{H}$ NMR $\left(400 \mathrm{MHz}, \mathrm{CD}_{3} \mathrm{OD}\right) \delta: 6.98 \sim 6.83(\mathrm{~m}$, $2 \mathrm{H}), 6.68 \sim 6.58(\mathrm{~m}, 1 \mathrm{H}), 5.94 \sim 5.84(\mathrm{~m}, 1 \mathrm{H}), 5.67 \sim 5.66$ $(\mathrm{m}, 1 \mathrm{H}), 5.12 \sim 4.99(\mathrm{~m}, 5 \mathrm{H}), 2.71 \sim 2.64(\mathrm{~m}, 2 \mathrm{H}), 2.51 \sim$ $2.45(\mathrm{~m}, 2 \mathrm{H}), 2.18 \sim 2.01(\mathrm{~m}, 4 \mathrm{H}), 1.63(\mathrm{~s}, 3 \mathrm{H}), 1.48(\mathrm{~s}$, $3 \mathrm{H}), 1.29(\mathrm{~m}, 1 \mathrm{H}), 1.23(\mathrm{~s}, 3 \mathrm{H}), 0.99(\mathrm{~s}, 3 \mathrm{H}) ;{ }^{13} \mathrm{C} \mathrm{NMR}$ (125 MHz, $\left.\mathrm{CD}_{3} \mathrm{OD}\right) \delta: 209.4,196.3,152.6,146.0,135.0$, $133.8,129.8,125.4,125.3,119.7,119.5,118.3,117.4$, 115.1, 52.2, 47.7, 40.7, 37.5, 32.3, 30.1, 27.2, 26.0, 23.2, 18.1; HRMS calcd for $\mathrm{C}_{29} \mathrm{H}_{34} \mathrm{O}_{6} \mathrm{Na}[\mathrm{M}+\mathrm{Na}]^{+}$501.2248, found 501.2246.

4-烯丙基-8-异戊烯基(代)山竹醇(3): 黄色粘稠物, $264.4 \mathrm{mg}$, 产率 58\%. ${ }^{1} \mathrm{H}$ NMR (400 MHz, CD $\left.\mathrm{OD}\right) \delta$ : $7.16 \sim 6.98(\mathrm{~m}, 2 \mathrm{H}), 6.68 \sim 6.59(\mathrm{~m}, 1 \mathrm{H}), 5.67 \sim 5.65(\mathrm{~m}$, $1 \mathrm{H}), 5.19 \sim 4.99(\mathrm{~m}, 4 \mathrm{H}), 2.72 \sim 2.64(\mathrm{~m}, 2 \mathrm{H}), 2.50 \sim 2.45$ $(\mathrm{m}, 2 \mathrm{H}), 2.18 \sim 2.01(\mathrm{~m}, 4 \mathrm{H}), 1.73(\mathrm{~s}, 3 \mathrm{H}), 1.66(\mathrm{~s}, 3 \mathrm{H})$, $1.63(\mathrm{~s}, 3 \mathrm{H}), 1.47$ (s, 3H), $1.29(\mathrm{~m}, 1 \mathrm{H}), 1.24(\mathrm{~s}, 3 \mathrm{H}), 0.99$ $(\mathrm{s}, 3 \mathrm{H}) ;{ }^{13} \mathrm{C} \mathrm{NMR}\left(125 \mathrm{MHz}, \mathrm{CD}_{3} \mathrm{OD}\right) \delta: 209.7,196.0$, $194.1,152.5,146.1,135.5,134.9,133.7,129.6,125.3$, $125.2,120.8,119.7,118.1,117.4,115.1,58.3,54.8,47.7$, 41.0, 32.2, 30.0, 27.2, 26.3, 26.0, 23.3, 18.2, 18.1; HRMS calcd for $\mathrm{C}_{29} \mathrm{H}_{34} \mathrm{O}_{6} \mathrm{Na}[\mathrm{M}+\mathrm{Na}]^{+}$529.2561, found 529.2560 .

\section{2 .11 细胞增殖分析}

MTT 法检测细胞增殖: 将对数生长期的 SCC-15 细 胞悬液调整浓度至 $1 \times 10^{4} / \mathrm{mL}$ 和 CAL-27 调至 $3 \times$ $10^{4} / \mathrm{mL}$ 后, 接种到 96 孔培养板, 每孔 $200 \mu \mathrm{L}$, 细胞贴壁 后, 根据预实验结果, 分别加入不同浓度的山竹醇和山 竹醇类似物(5、10、20、30、40、50 $\mu \mathrm{mol} \cdot \mathrm{L}^{-1}$ ). 每个浓 度设置 6 个复孔, 同时设置不加细胞的调零孔和不加药 物的阴性对照孔. 在 $37{ }^{\circ} \mathrm{C} 5 \% \mathrm{CO}_{2}$ 分别孵育 24、48、 $72 \mathrm{~h}$, 每孔加入 $20 \mu \mathrm{L} \mathrm{MTT}$ 溶液 $(5 \mathrm{mg} / \mathrm{mL})$, 继续培养 4 $\mathrm{h}$ 后终止培养, 吸去孔内培养液, 每孔加入 $200 \mu \mathrm{L}$ 二甲 基亚砜, 置摇床上低速振荡 $10 \mathrm{~min}$, 使结晶物充分溶解. 在酶联免疫检测仪波长 OD490 nm 处测量各孔的吸光 值. 应用 SPSS 17.0 for windows 统计软件进行统计分析. 采用 ANOVA 检验, 以 $\alpha=0.05$ 作为检验水准.

\subsubsection{2 分子对接模拟}

首先在 PDB (http://www.rcsb.org)蛋白质数据库下
载 5-LOX(PDB_ID: 3V99)的晶体结构. 借助软件 DS 2019 对其进行去除水分子, 去除蛋白质多构象, 补充非 完整的氨基酸残基, 为蛋白加氢等预处理. 经过处理的 蛋白, 可进行后续的对接等操作. 接着借助软件 DS 2019，对小分子配体进行预处理. 最后利用处理好的蛋 白和配体进行分子对接，使用 DS 2019 软件中的 CDOCKER 模块, 设置相应的流程参数, 运行分子对接 模拟.

辅助材料(Supporting Information) 目标化合物的 ${ }^{1} \mathrm{H}$ $\mathrm{NMR},{ }^{13} \mathrm{C} \mathrm{NMR}$ 谱图. 这些材料可以免费从本刊网站 (http://siocjournal.cn/)上下载.

\section{References}

[1] Newman, D. J.; Cragg, G. M.; Snader, K. M. J. Nat. Prod. 2003, 66, 1022.

[2] Aggarwal, B. B.; Kunnumakkara, A. B. Molecular Targets and Therapeutic Use of Spices, World Scientific Publishing Co. Pte. Ltd., 2009, pp. 281 309.

[3] Schobert, R.; Biersack, B. Chem. Biodiversity. 2019, 16, e1900366.

[4] Aggarwal, S.; Das, S. N. Tumor. Biol. 2016, 37, 7175.

[5] Ranjbarnejad, T.; Saidijam, M.; Tafakh, M. S.; Pourjafar, M.; Talebzadeh, F.; Najafi, R. Hum. Exp. Toxicol. 2017, 36, 692.

[6] Li, F.; Shanmugam, M. K.; Chen, L. X.; Chatterjee, S.; Basha, J.; Kumar, A. P.; Kundu, T. K.; Sethi, G. Cancer Prev. Res. (Phila) 2013, 6, 843 .

[7] Wang, J. H.; Wang, L. W..; Ho, C. T.; Zhang, K. S.; Liu, Q.; Zhao, H. J Agric. Food Chem. 2017, 65, 3675.

[8] Ahmad, A.; Wang, Z. W.; Wojewoda, C.; Ali, R.; Kong, D.; Maitah, M. Y.; Banerjee, S.; Bao, B.; Padhye, S.; Sarkar, F. H. Front. Biosci. $1492,3,1483$.

[9] Liu, C.; Ho, P. C.; Wong, F. C.; Sethi, G.; Wang, L. Z.; Goh, C. Cancer Lett. 2015, 362, 8.

[10] Liao, C. H.; Ho, C. T.; Lin, J. K. Biochem. Biophys. Res. Commun. $\mathbf{2 0 0 5}, 329,1306$.

[11] Liao, C. H.; Sang, S. M.; Liang, Y. C.; Ho, C. T.; Lin, J. K. Mol. Carcinog. 2004, 41, 140.

[12] Zhou, X. Y.; Cao, J.; Han, C. M.; Li, S. W.; Zhang, C.; Du, Y, D.; Zhou, Q. Q.; Zhang, X. Y.; Chen, X. Bioorg. Chem. 2017, 71, 74.

[13] Cao, J.; Han, C. M.; Zhang, G. L.; Zhou, X. Y.; Li, S. W.; Du, Y. D.; Zhao, S.; Zhang, X. Y.; Chen, X. Chin. J. Org. Chem. 2017, 37, 8 (in Chinese).

(曹菁，韩超明，张桂莲，周新芗，李舒雯，杜银端，赵帅，张辛 燕, 陈新, 有机化学, 2017, 37, 8.)

[14] Han, C. M.; Zhou, X. Y.; Cao, J.; Zhang, X. Y.; Chen, X. Bioorg. Chem. 2015, 60, 123.

[15] Chen, X.; Zhang, X. Y.; Lu, Y.; Shim, J. Y.; Sang, S. M.; Sun, Z.; Chen, X. X. Nutr. Cancer. 2012, 64, 1211.

[16] Socolsky, C.; Plietker, B. Chem.-Eur. J. 2015, 21, 3053.

[17] Guttroff, C.; Baykal, A.; Wang, H. H.; Popella, P.; Kraus, F.; Biber, N.; Krauss, S.; Gotz, F.; Plietker, B. Angew. Chem., Int. Ed. 2017, $56,15852$.

[18] Biber, N.; Mows, K.; Plietker, B. Nat. Chem. 2011, 3, 938.

[19] Holzwarth, M.; Dieskau, A.; Tabassam, M.; Plietker, B. Angew. Chem. Int. Ed. 2009, 48, 7251.

[20] Saba, S.; Brescia, A.; Kaloustian, M. Tetrahedron Lett. 1991, 32, 5031.

(Li, L.; Fan, Y.) 